\title{
Inflammation drives thrombosis after Salmonella infection via CLEC-2 on platelets
}

\author{
Jessica R. Hitchcock, ${ }^{1}$ Charlotte N. Cook, ${ }^{1}$ Saeeda Bobat, ${ }^{1}$ Ewan A. Ross, ${ }^{1}$ Adriana Flores-Langarica, ${ }^{1}$ Kate L. Lowe, ${ }^{2}$ \\ Mahmood Khan, ${ }^{1}$ C. Coral Dominguez-Medina, ${ }^{1}$ Sian Lax, ${ }^{1}$ Manuela Carvalho-Gaspar, ${ }^{1}$ Stefan Hubscher, ${ }^{1}$ C. Ed Rainger, ${ }^{2}$ \\ Mark Cobbold, ${ }^{1}$ Christopher D. Buckley, ${ }^{1}$ Tim J. Mitchell, ${ }^{1}$ Andrea Mitchell, ${ }^{1}$ Nick D. Jones, ${ }^{1}$ N. Van Rooijen, ${ }^{3}$ Daniel Kirchhofer, ${ }^{4}$ \\ Ian R. Henderson, ${ }^{1}$ David H. Adams, ${ }^{1}$ Steve P. Watson, ${ }^{2}$ and Adam F. Cunningham ${ }^{1}$ \\ 1MRC Centre for Immune Regulation, Institute of Immunology and Immunotherapy and Institute for Microbiology and Infection, and ${ }^{2}$ Institute of Cardiovascular Sciences, \\ College of Medical and Dental Sciences, University of Birmingham, Birmingham, United Kingdom. ${ }^{3}$ Department of Molecular Cell Biology, Faculty of Medicine, Vrije Universiteit, Amsterdam, Netherlands. \\ ${ }^{4}$ Department of Early Discovery Biochemistry, Genentech Inc., South San Francisco, California, USA.
}

\begin{abstract}
Thrombosis is a common, life-threatening consequence of systemic infection; however, the underlying mechanisms that drive the formation of infection-associated thrombi are poorly understood. Here, using a mouse model of systemic Salmonella Typhimurium infection, we determined that inflammation in tissues triggers thrombosis within vessels via ligation of C-type lectin-like receptor-2 (CLEC-2) on platelets by podoplanin exposed to the vasculature following breaching of the vessel wall. During infection, mice developed thrombi that persisted for weeks within the liver. Bacteria triggered but did not maintain this process, as thrombosis peaked at times when bacteremia was absent and bacteria in tissues were reduced by more than $\mathbf{9 0 \%}$ from their peak levels. Thrombus development was triggered by an innate, TLR4-dependent inflammatory cascade that was independent of classical glycoprotein VI-mediated (GPVI-mediated) platelet activation. After infection, IFN- $\gamma$ release enhanced the number of podoplanin-expressing monocytes and Kupffer cells in the hepatic parenchyma and perivascular sites and absence of TLR4, IFN- $\gamma$, or depletion of monocytic-lineage cells or CLEC-2 on platelets markedly inhibited the process. Together, our data indicate that infection-driven thrombosis follows local inflammation and upregulation of podoplanin and platelet activation. The identification of this pathway offers potential therapeutic opportunities to control the devastating consequences of infection-driven thrombosis without increasing the risk of bleeding.
\end{abstract}

\section{Introduction}

Thrombosis-associated events are among the leading causes of death worldwide. Systemic infections caused by a plethora of bacterial genera can initiate thrombus development. While the mechanisms that underlie this process during some infections, particularly for Gram-positive organisms such as staphylococci or streptococci, have been described, these mechanisms are not universally applicable. This is illustrated by the limited efficacy of current treatments to modulate thrombosis during infection.

In typhoid, a systemic infection caused by Salmonella Typhi, bacteria colonize sites such as the spleen and liver, where they reside within monocytes and macrophages $(1,2)$. This systemic spread of infection causes a modest bacteremia, typically with less than 10 bacteria per $\mathrm{ml}$ of blood, and a widespread inflammatory response $(3,4)$. The infection is limited by the innate immune system, but control and clearance of the bacteria require an adaptive Th1 response and induction of IFN- $\gamma(5)$. Before the introduction of antimicrobial treatments for typhoid, thrombosis was commonly described as a complication of this infection that could result in death; reports have noted a relationship between typhoid and thrombosis for over a century (6-8). Thrombosis is also seen in mouse models of typhoid caused by Salmonella Typhimurium,

Conflict of interest: The authors have declared that no conflict of interest exists. Submitted: September 17, 2014; Accepted: October 8, 2015.

Reference information: / Clin Invest. 2015;125(12):4429-4446. doi:10.1172/JCI79070. suggesting that this consequence of systemic infection is conserved in multiple species (9). Susceptibility of mice to Salmonella infections is strongly influenced by their expression of distinct versions of natural resistance-associated macrophage protein 1 (NRAMP1, also known as SLC11A1) (10), which differ by a single amino acid. When mice that are innately more resistant to virulent Salmonella are infected, thrombi can be seen in multiple sites throughout the body, including the spleen, liver, and kidneys (9, 11). On the other hand, mice that, due to Nramp1, are hypersusceptible to systemic infection with virulent $S$. Typhimurium, such as the commonly used laboratory strains C57BL/6J and BALB/c, succumb rapidly to this infection in just a few days. This adds a layer of complexity in mechanistic studies, since most genetically altered mice are of a C57BL/6J or BALB/c background. Nevertheless, many features of $S$. Typhimurium infection in resistant mice, including the modest bacteremia and thrombosis in the liver, can be reproduced in hypersusceptible mice using an attenuated strain of $S$. Typhimurium. In such experimentally infected mice, bacterial numbers can be in the hundreds of thousands in sites such as the spleen and liver and yet be concurrently many thousand-fold lower in the blood before the infection resolves gradually over a period of weeks (12). Infection of resistant mice with virulent Salmonella or infection of hypersusceptible mice with attenuated Salmonella both commonly result in thrombosis $(9,13)$.

Recently, a beneficial role for thrombosis during inflammation has been described, whereby platelet aggregation and coagulation 


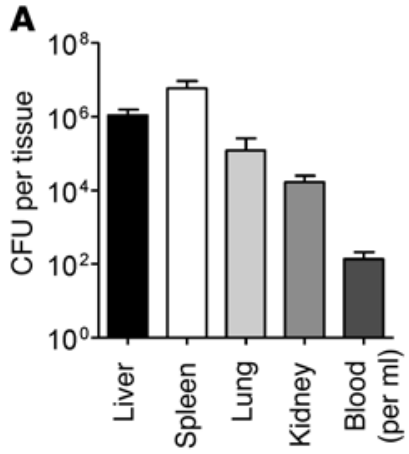

D
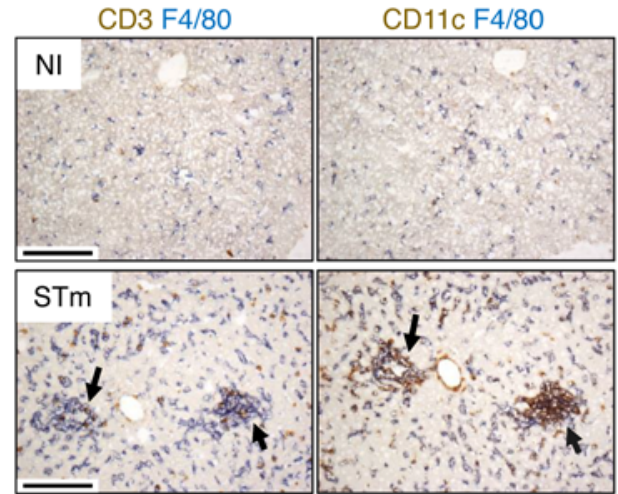

B

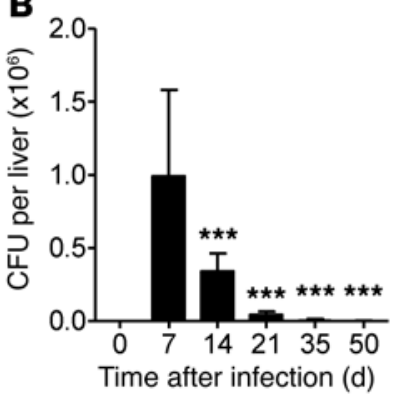

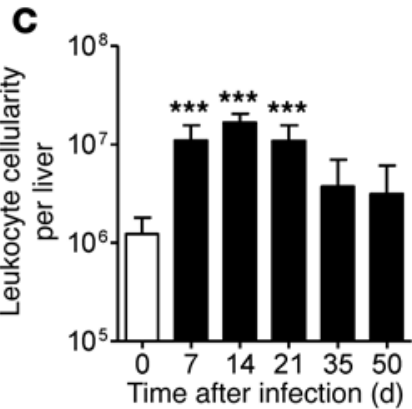

G

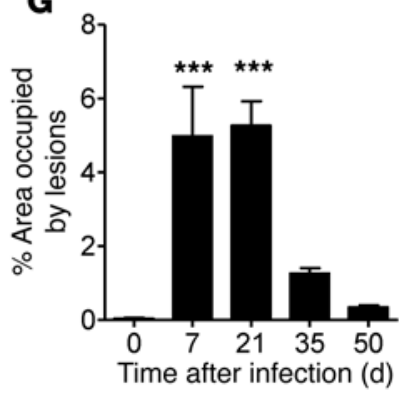

E

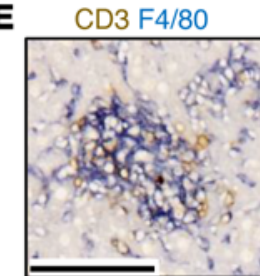

CD3 Ly6C

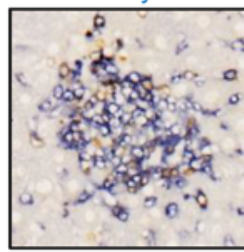

CD11c F4/80

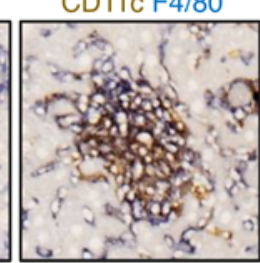

CD3 Ly6G

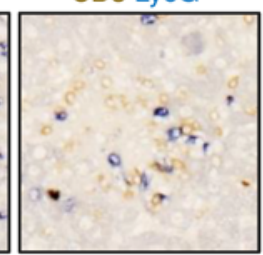

F

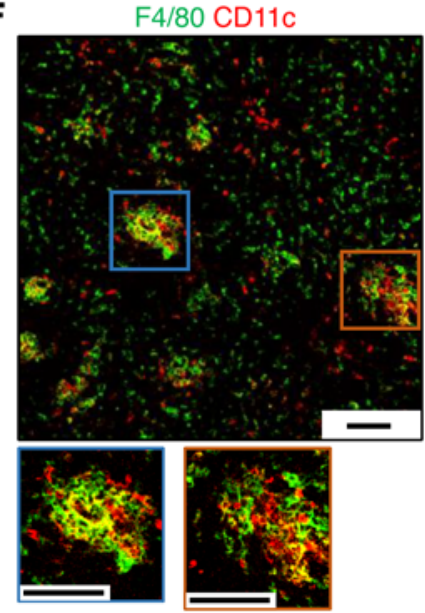

Figure 1. Inflammatory foci develop in the liver during S. Typhimurium infection. WT mice were infected i.p. with $5 \times 10^{5} \mathrm{CFU}$ S. Typhimurium (STm). (A) Bacterial colonization 7 days after infection. (B) Bacterial colonization of the liver. (C) Number of leukocytes isolated from the liver. (D) Noninfected (NI) and day 7-infected serial liver sections stained by IHC for F4/80 (blue), CD3 (brown); and F4/80 (blue), CD11c (brown). Arrows indicate inflammatory lesions. (E) Serial sections of a lesion stained for F4/80 (blue), CD3 (brown); F4/80 (blue), CD11c (brown); Ly6C (blue), CD3 (brown); and Ly6C (blue), CD3 (brown). (F) Fluorescent confocal microscopy image of day 7-infected liver stained for F4/80 (green) and CD11c (red). (C) Percentage area of liver occupied by inflammatory lesions quantified by point counting. Graphs show mean $+\mathrm{SD}$. Data are representative of a minimum of 3 experiments, where $n \geq 4$ mice in each group. Statistical significance (1-way ANOVA with Dunnett's test) was determined relative to day 7 after infection (B) or relative to noninfected mice (C and $\mathbf{G}) .{ }^{* *} P \leq 0.001$. Scale bars: $100 \mu \mathrm{m}$.

can contribute to bacterial containment and ultimately clearance within the vasculature at localized sites, a process termed immunothrombosis (14). Indeed, some bacteria actively dissociate clots, enabling bacterial dissemination. For example, streptococci species can dissolve fibrin via streptokinase-mediated plasmin activation (15). Additionally, neutrophil extracellular traps, which also contribute to bacterial containment within the vasculature, exhibit potent procoagulant features $(16,17)$. On the other hand, certain bacteria purposely activate coagulation as a method of immune evasion. For example, Staphylococcus aureus disguises itself within a fibrin-containing pseudocapsule formed via staphylocoagulasemediated prothrombin activation (18). Furthermore, direct interaction between bacteria and platelets, which may also contribute to the widely acknowledged ability of bacteria to manipulate the clotting system, has been described (19-21). Therefore, although bacteria can have a profound relationship with the clotting cascade, the actual nature of this is variable and often complex.

Despite being the focus of much investigation, the link between the distinct pathways of inflammation and platelet activation is not yet fully understood. Platelets can be activated through multiple pathways, including by ligation of platelet-expressed C-type lectinlike receptor-2 (CLEC-2) to its cognate ligand/receptor, podoplanin
(22). In the steady state, podoplanin has a restricted distribution outside the vasculature, with high levels on lymphatic endothelial cells, but is markedly upregulated on multiple cells, including macrophages and Th17 cells, during inflammation $(23,24)$.

In this study, we investigate the molecular mechanism of thrombus development in the hepatic vasculature during systemic $S$. Typhimurium infection. We show that inflammation directly induces thrombosis and that these formerly distinct host responses are linked by podoplanin upregulation on macrophage subsets in the liver during infection. This results in an alternative pathway of in vivo platelet activation during inflammation, mediated by CLEC-2 and its ligand, podoplanin. This distinct mechanism of platelet activation and thrombosis is independent of the major hemostatic pathways. This therefore identifies new opportunities for therapeutic interventions during inflammation-associated thrombosis that are likely to be unaffected by the deleterious bleeding complications associated with current antiplatelet strategies.

\section{Results}

Systemic S. Typhimurium infection induces thrombi formation within the hepatic vasculature. Administration i.p. of attenuated $S$. Typhimurium resulted in a systemic infection characterized by 
A
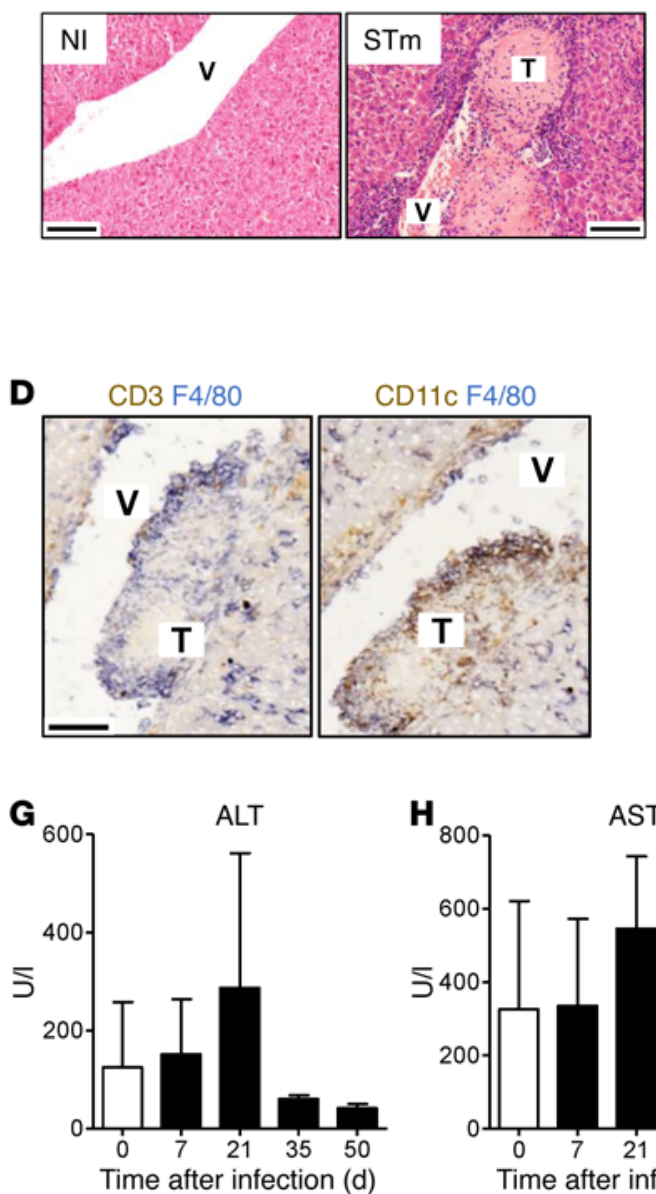

B

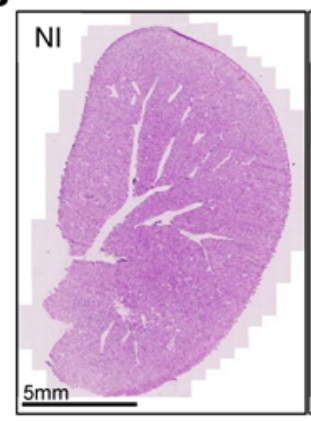

E

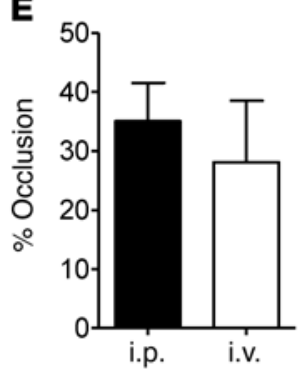

I

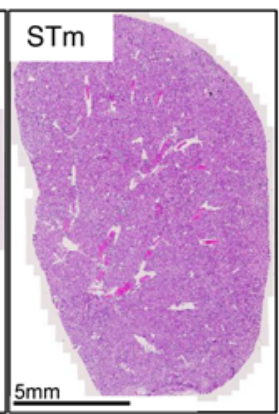

$\mathbf{F}$

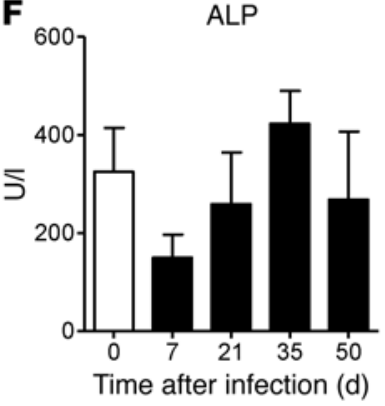

ALP

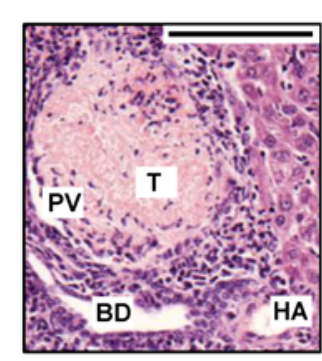

Figure 2. Extensive thrombosis is a consequence of $\mathbf{S}$. Typhimurium infection. WT mice were infected i.p. with S. Typhimurium as above. (A) H\&E staining of paraffin-embedded sections from noninfected and day 7-infected liver. (B) Tile scan of H\&E-stained frozen liver sections from noninfected and day 7-infected mice. (C) Thrombi are observed in the portal vein; 7 day-infected paraffin-embedded section. (D) IHC staining of frozen liver sections at day 7 after infection: CD3 (brown), F4/80 (blue); and CD11c (brown), F4/80 (blue). (E) WT mice were infected i.p. or i.v. as above for 7 days. Thrombosis was examined by $\mathrm{H} \& \mathrm{E}$, and percentage of vessel occlusion was quantified in large vessels. (F-J) WT mice were infected i.p. as above, and enzymes associated with liver injury were measured in the serum throughout infection. Graphs show mean + SD. Data are representative of a minimum of 3 experiments, where $n \geq 4$ mice in each group. Scale bars: $100 \mu \mathrm{m}$. V, blood vessel; T, thrombus; PV, portal vein; HA, hepatic artery; BD, bile duct; ALP, alkaline phosphatase; ALT, alanine aminotransferase; AST, aspartate aminotransferase.

multiple sites of bacterial colonization, including spleen, liver, kidney, and lungs (Figure 1A). In the liver, bacterial load peaked within 7 days, and bacteria were largely cleared from this site after 1 month (Figure 1B). Infection induced the accumulation of leukocytes throughout the liver, which were organized in discrete inflammatory foci (Figure 1, C and D). Foci were primarily composed of myeloid cells that express varying combinations of $\mathrm{F} 4 / 80$, CD11c, and Ly6C (primarily cells of the monocyte lineage) as well as a small number of $\mathrm{CD}^{+} \mathrm{T}$ lymphocytes, but few $\mathrm{Ly} 6 \mathrm{G}^{+}$neutrophils (Figure 1, E and F). Inflammatory foci were still present at 21 days, when bacterial numbers were significantly reduced (Figure $1 G)$. Thus, the host inflammatory response to systemic infection persisted in the liver once bacterial clearance was established.

In addition to the development of inflammatory foci in the liver, $S$. Typhimurium infection induced extensive thrombosis within the liver vasculature (Figure 2, A and B). Thrombi formed predominantly within branches of the portal vein (as identified by the adjacent vessels of the portal triad) and were not detected in arterial vessels or sinusoids (Figure 2C). In addition, thrombi typically associated with perivasculature inflammatory infiltrates and were frequently surrounded by a peripheral cuff of leukocytes, suggesting thrombi formed in situ in the liver (Figure 2D). Thrombosis development was similar when bacteria were administered by either an i.p. or an i.v. route (Figure 2E). Despite the extensive accumulation of leukocytes and the development of thrombi, biomarkers of liver injury showed limited changes; there was indication of some reversible injury, which returned to preinfection levels by day 35 after infection (Figure 2, F-J).

Platelet thrombi develop in parallel with inflammatory lesions. Thrombi were white upon macroscopic inspection and were thus primarily composed of platelets, as confirmed using a plateletspecific marker, the integrin subunit $\alpha \mathrm{IIb} / \mathrm{CD} 41$ (Figure 3, A and B). Thrombi were detectable by day 5 after infection and peaked in extent between days 7 and 21 (Figure 3C and data not shown). 
A

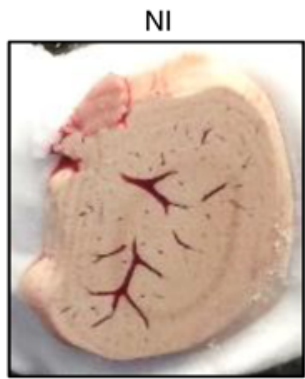

STm

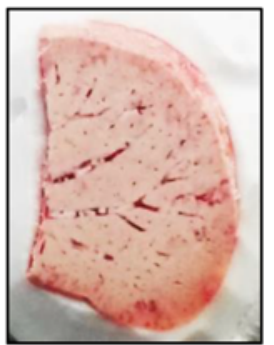

B

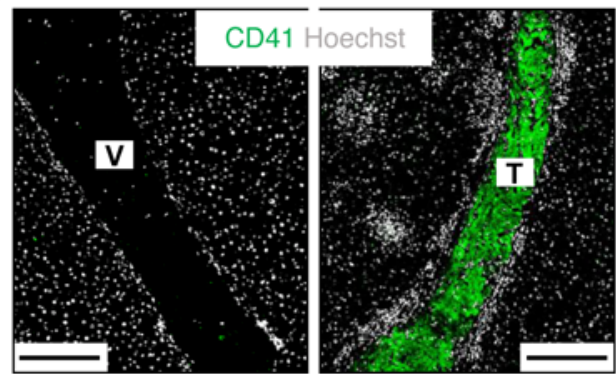

E

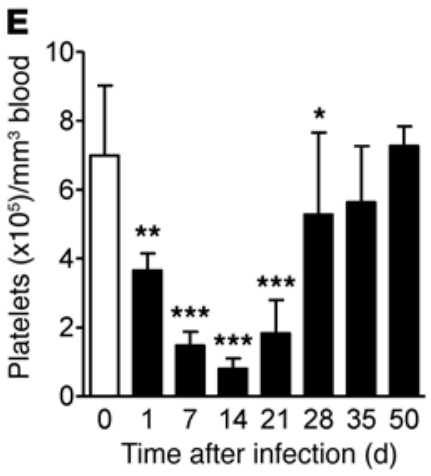

$\mathbf{F}$
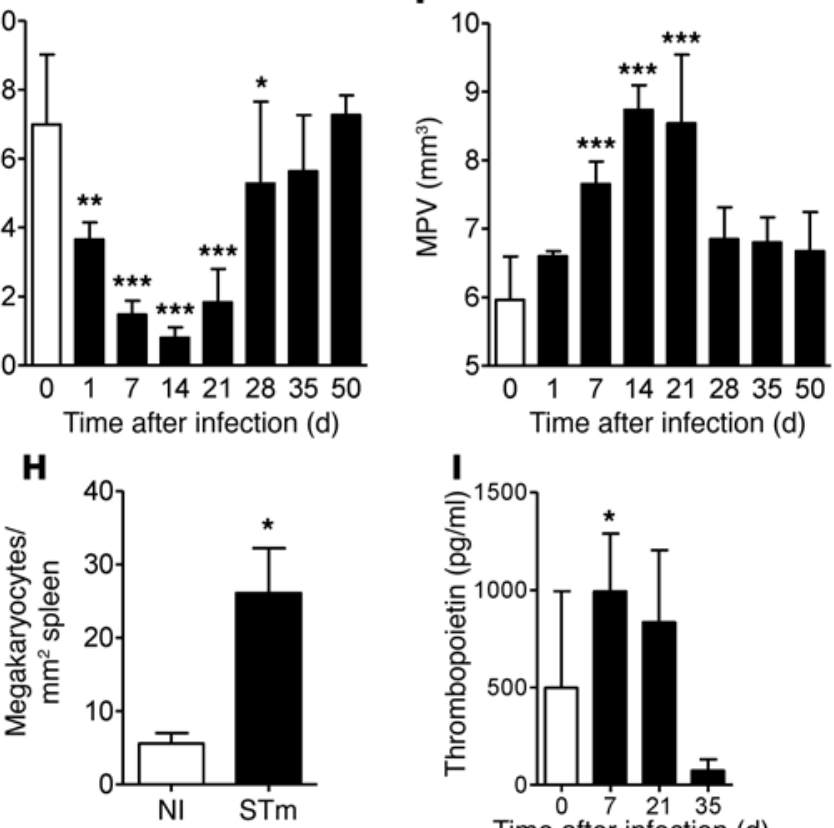

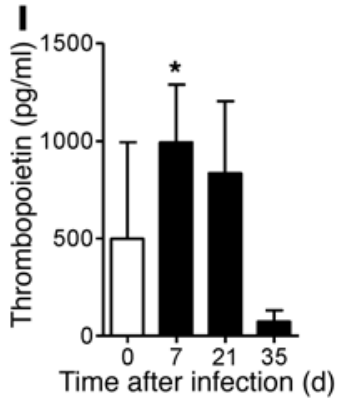

Figure 3. Infection is associated with altered platelet homeostasis. WT mice were infected i.p. as above. (A) Photomicrographs of noninfected and day 7-infected frozen liver tissue. (B) Fluorescent confocal microscopy on frozen liver sections of noninfected and day 7-infected mice: CD41 (green), Hoechst (gray). Scale bars: $200 \mu \mathrm{m}$. (C) Vessel occlusion was quantified by point counting of large vessels per tissue section. Statistical significance of thrombosis resolution was determined relative to day 7 after infection. (D) Bacterial colonization of the blood was enumerated at the indicated time points after infection. (E) Platelet numbers and (F) mean platelet volume (MPV) in the blood throughout infection. (G) Megakaryocytes (indicated by circles) in the spleen; H\&E staining of frozen spleen sections at days 0 and 7 after infection. F, follicle. Scale bar: $100 \mu \mathrm{m}$. (H) Megakaryocytes per mm² spleen. (I) Thrombopoietin concentration in the serum at the indicated time points after infection. Graphs show mean $+\mathrm{SD}$. In all cases, data shown are representative of a minimum of 3 experiments, where $n \geq 4$ mice in each group. Statistical significance was determined relative to day 7 after infection or to noninfected mice (D-F, $\mathbf{H}$, and $\mathbf{I}) .{ }^{*} P \leq 0.05 ;{ }^{* *} P \leq 0.01 ;{ }^{* *} P \leq 0.001$, 1-way ANOVA with Dunnett's test (C-F and $\mathbf{I}$ ) or Mann-Whitney sum of ranks test (H).

However, while the development of thrombi was infection mediated, the continued presence of thrombosis was not dependent upon a continuing bacteremia (Figure 3D). In addition to an absence of bacteremia beyond day 14, tissue bacterial loads were cleared to approximately $10 \%$ of their peak numbers by day 21 , when vessel occlusion was still near its peak (Figure 1B and Figure 3C). Thus, $S$. Typhimurium induces thrombosis within the liver, but the maintenance of this thrombosis is not determined by the persistence of bacteria in the blood or in the tissues.

Thrombosis is mirrored by a severe thrombocytopenia, a finding commonly observed with this infection (Figure $3 \mathrm{E}$ and ref. 9). Mean platelet volume and megakaryocyte numbers in the spleen, which are commonly associated with thrombocytopenia, were also increased, and the marker of accelerated platelet production, serum thrombopoietin, was increased during infection (Figure 3, F-I, and ref. 25). Thus, diminished platelet count and 2 indirect markers of increased platelet production, mean platelet volume and extramedullary megakaryocytopoiesis, parallel the kinetics of thrombosis severity.

Thrombus development is not altered by common antiplatelet agents. One prominent mechanism of initiation of platelet-driven thrombosis is through activation of the platelet-specific collagen receptor glycoprotein VI (GPVI) (26). However, thrombus development during $S$. Typhimurium infection was not altered in mice deficient in GPVI, indicating that thrombosis must be regulated by an alternative pathway that is specifically induced after exposure to bacteria (Figure 4, $\mathrm{A}$ and B). Furthermore, treatment of mice with the common antiplatelet agent clopidogrel, which targets the platelet ADP receptor P2 ${ }_{12}$, also had no effect on thrombus development, and neither did treatment with aspirin or heparin (Figure 4, C-F).

The observation that the dynamics of induction, maintenance, and resolution of thrombi parallel those of inflammatory lesions 
A

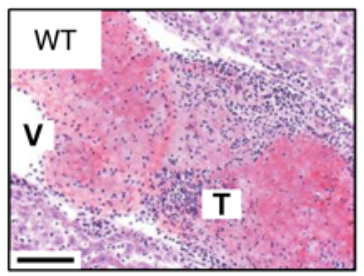

C

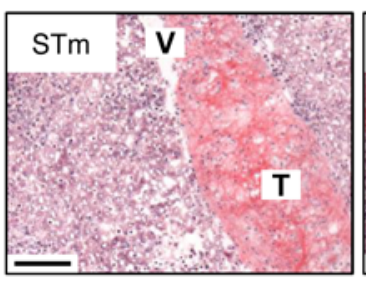

$\mathbf{F}$

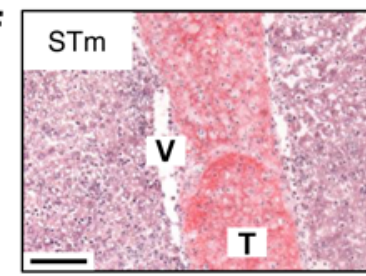

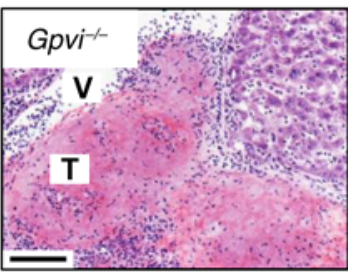

B

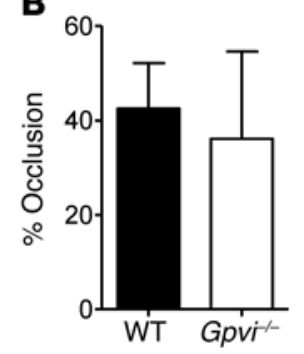

E

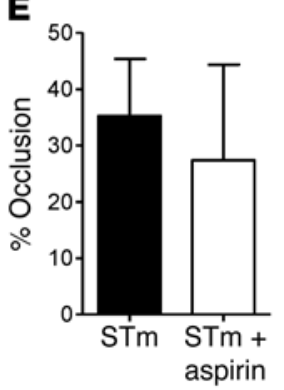

H

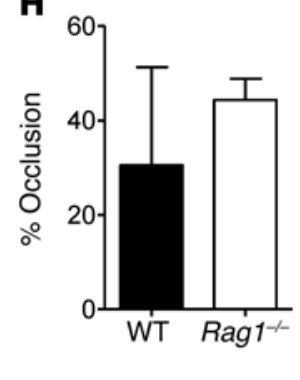

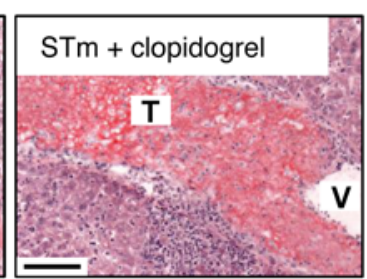
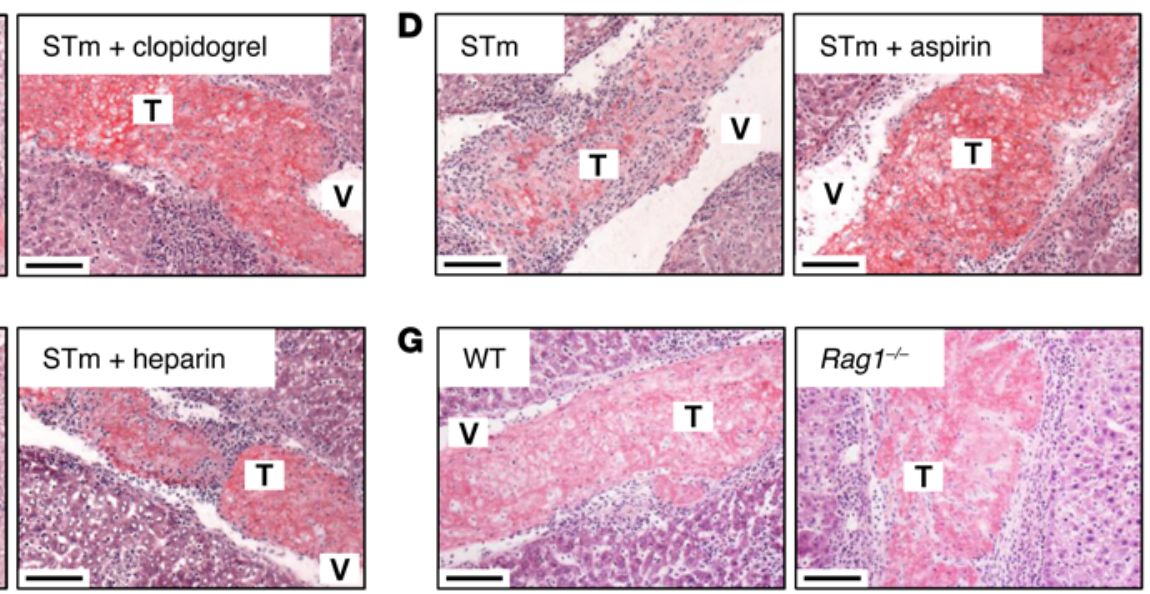

Figure 4. Thrombus development is not altered by common antiplatelet agents. (A) WT and Gpvi-/- mice were infected i.p. as above for 7 days. Thrombi were examined by H\&E and (B) quantified by point counting of large vessels per tissue section. (C) WT mice were treated with clopidogrel (50 mg/kg i.v.) on day 3 after infection and daily $(15 \mathrm{mg} / \mathrm{kg}$ i.v.) thereafter; thrombi were examined at day 7. (D) WT mice were treated with aspirin (30 mg/l in drinking water) on day 5 after infection and daily thereafter; thrombi were examined after 13 days and (E) were quantified by point counting as above. (F) WT mice were treated daily with heparin sodium (100 U/kg i.v.) from day 3 after infection; thrombi were examined at day 7 after infection. (G) WT and Rag $1^{-/-}$mice were infected i.p as above; thrombi were examined after 7 days and $(\mathbf{H})$ were quantified as above. All H\&E was performed on frozen liver sections; all images are representative. Scale bars: $100 \mu \mathrm{m}$. Graphs show mean + SD. Experiments were performed at least twice with each group containing $\geq 4$ mice.

led us to hypothesize that hepatic inflammation and thrombosis may be coregulated. If so, the link must be mediated through the innate immune system and not the adaptive system, since infected Rag1/- mice, which lack mature B and T lymphocytes, exhibit a level of thrombosis similar to that of WT mice at day 7 after infection (Figure $4, \mathrm{G}$ and $\mathrm{H}$ ).

TLR 4 and IFN- $\gamma$ mediate inflammation and thrombus development. A key sentinel molecule of the innate system that initiates inflammation in response to Gram-negative bacteria such as Salmonella is TLR4 (27). This receptor recognizes a range of bacterial antigens, including LPS or endotoxin, and is expressed on multiple cell types, including Kupffer cells and monocytes (28). S. Typhimurium infection of $\mathrm{Tlr} 4^{-/}$mice for 7 days did not induce inflammatory foci and thrombus development (Figure 5, A-C). Furthermore, thrombocytopenia was not detected in these mice after infection (Figure 5, D and E). However, TLR4 stimulation after challenge of WT mice for 7 days with $20 \mu \mathrm{g}$ of LPS alone was insufficient for thrombus development, demonstrating that thrombosis requires infection and is not merely associated with exposure to bacterial LPS (Figure 5F).

The absence of inflammation during early $S$. Typhimurium infection has been previously reported in IFN- $\gamma$-deficient mice (29). Paralleling an absence of inflammatory foci in Ifng $\mathrm{f}^{--}$mice at day 7, there was also a lack of thrombus development in these mice (Figure 5, G-I). Moreover, platelet counts and mean platelet vol- ume in Ifng ${ }^{-/}$mice were higher and lower, respectively, than those of infected WT animals and resembled those of the noninfected group (Figure 5, J and $\mathrm{K}$ ). The markedly diminished leukocyte accumulation after infection in both TLR4- and IFN- $\gamma$-deficient mice establishes a critical role for both mediators in initiating the inflammatory cascade (Figure 5, L and M). The absence of inflammation and thrombosis within the liver was independent of changes in bacterial burdens, which were similar or higher than in WT mice in each case (Figure 5, $\mathrm{N}$ and $\mathrm{O}$ ). This further supports the concept that bacterial infection is necessary to trigger the inflammatory and thrombotic cascades, but that additional mechanisms contribute to their maintenance.

Leukocytes isolated from day 7-infected Tlr $4^{-/}$livers cultured ex vivo produced considerably less IFN- $\gamma$ than those cultured from WT mice (Figure 5P), demonstrating the necessity of TLR4 signaling for optimal IFN- $\gamma$ production during infection. Therefore, thrombosis is induced by inflammation mediated by TLR4 ligation and IFN- $\gamma$ production.

Podoplanin expression is elevated during infection. Given the temporal relationship of inflammation and thrombosis, we sought to identify a common pathway that could link inflammation to platelet activation. One protein that is associated with both activities is podoplanin. This molecule is upregulated on a variety of cell types during inflammation and mediates platelet activation via platelet-expressed CLEC-2 $(30,31)$. To identify a potential role 

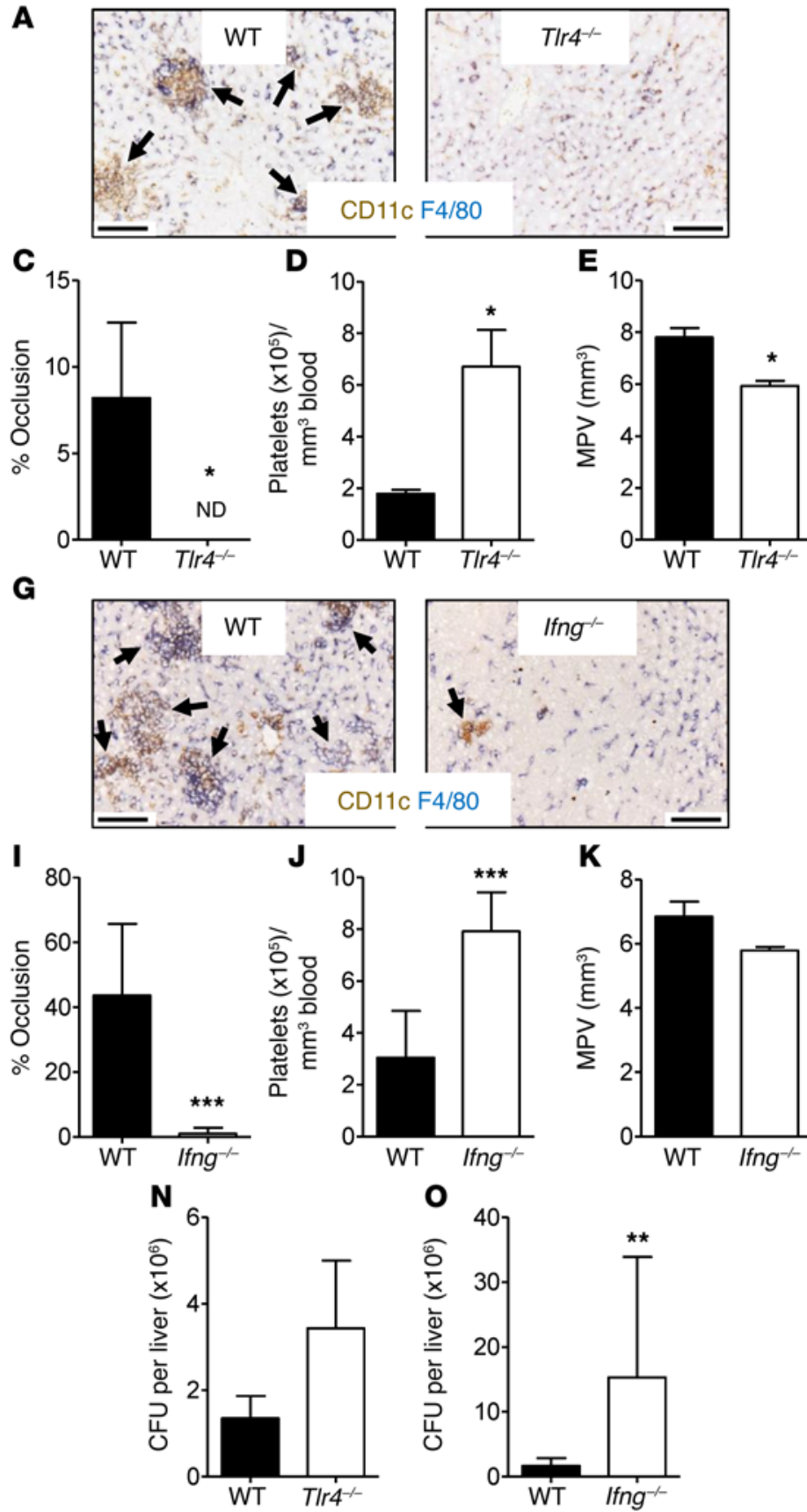

B
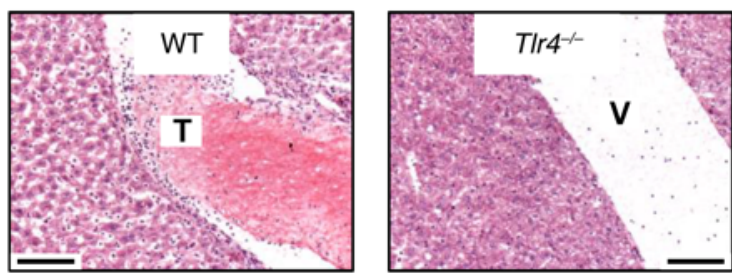

F
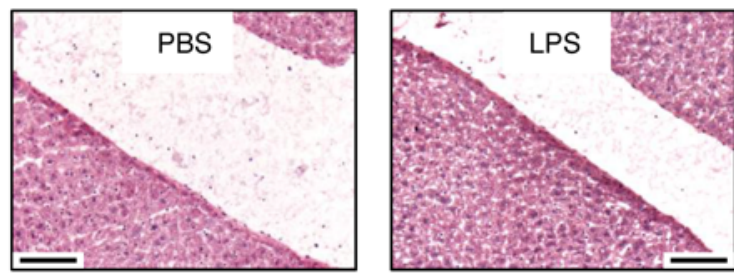

H

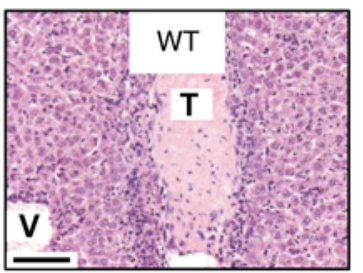

$\mathbf{L}$
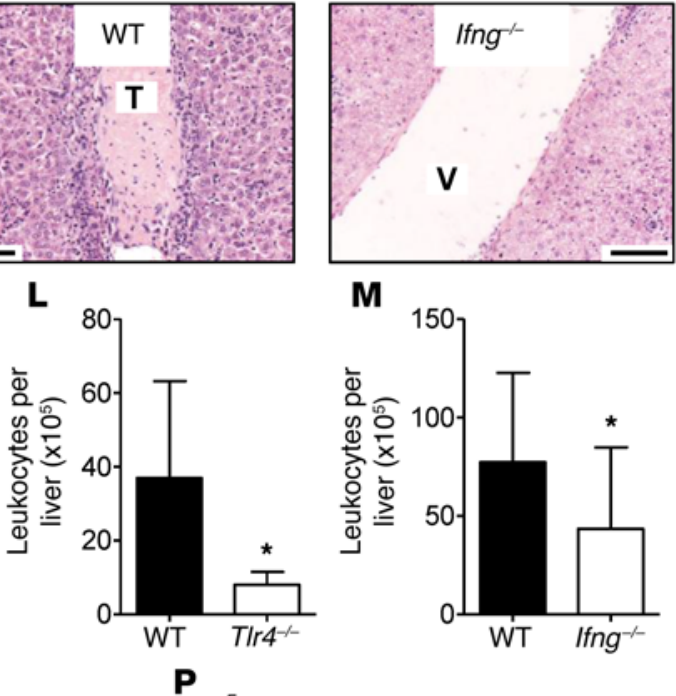

M
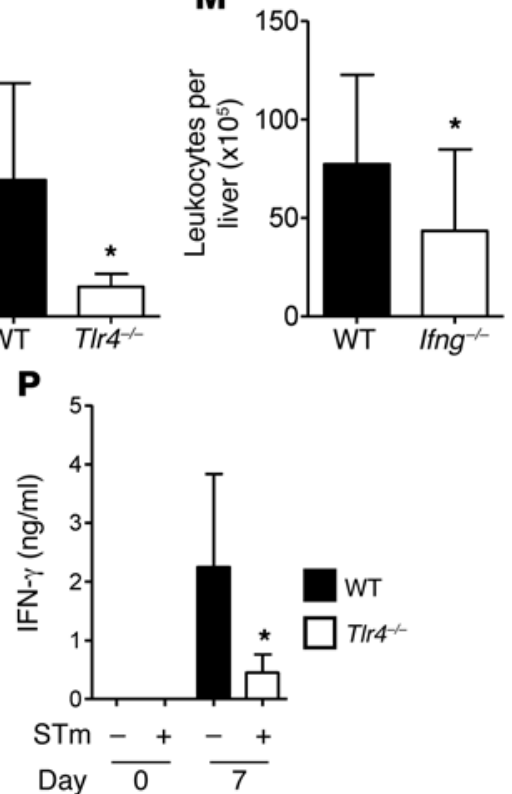

Figure 5. TLR4 and IFN- $\gamma$ mediate inflammation and thrombus development. WT and T/r4 ${ }^{-/-}$mice were infected i.p. as above for 7 days. (A) Leukocyte infiltration of the liver was examined by IHC: CD11C (brown), F4/80 (blue). (B) Thrombus development was assessed by H\&E staining and (C) was quantified by point counting of large vessels per tissue section. (D) Platelet numbers and (E) mean platelet volume were determined in the blood. (F) WT mice were treated with 20 $\mu \mathrm{g}$ LPS, and thrombus development was examined in the liver after 7 days. WT and Ifng ${ }^{-1-}$ mice were infected as above for 7 days, and (G) leukocyte infiltration of the liver was examined by IHC: CD11c (brown), F4/80 (blue). (H) Thrombus development was assessed by H\&E staining and (I) was quantified as above. (J) Platelet numbers and (K) mean platelet volume were determined in the blood. Number of leukocytes isolated from the livers of infected WT and (L) TIr4 ${ }^{-/-}$or (M) Ifng ${ }^{-1-}$ mice and ( $\mathbf{N}$ and $\mathbf{~}$ ) bacterial colonization of the liver were enumerated. (P) WT and TIr $4^{-/-}$mice were infected as above for 7 days, and leukocytes isolated from livers were cultured for 48 hours. Secreted IFN- $\gamma$ was measured in culture supernatants by ELISA. Graphs show mean + SD. Experiments were performed at least twice, with each group containing $\geq 4$ mice. Statistical significance was determined relative to WT mice. ${ }^{*} P \leq 0.05 ;{ }^{* *} P \leq 0.01 ;{ }^{* * *} P \leq 0.001$, Mann-Whitney sum of ranks test. ND, not detectable. All images are representative; black arrows indicate inflammatory lesions. Scale bars: $100 \mu \mathrm{m}$.

for podoplanin during $S$. Typhimurium infection, we monitored its expression in the liver by histology and flow cytometry. Podoplanin expression was mainly restricted to lymphatic endothelial cells in noninfected mice (Figure 6A). Expression was dramatical- ly increased in the liver within 24 hours of infection and remained elevated throughout infection (Figure 6A and Supplemental Figure 1; supplemental material available online with this article; doi:10.1172/JCI79070DS1). Vascular endothelial $\left(\mathrm{CD} 31^{+}\right)$cells 
A
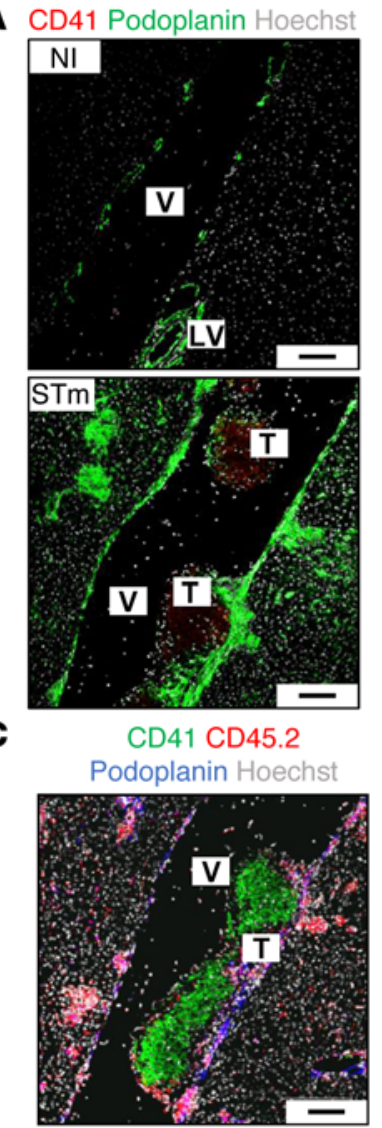

B
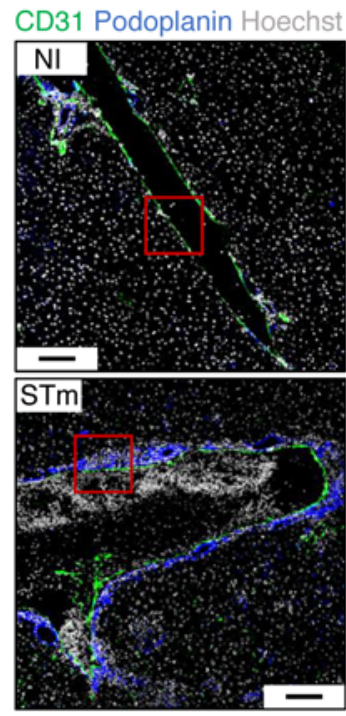

D

CD31 CD248 Podoplanin Hoechst

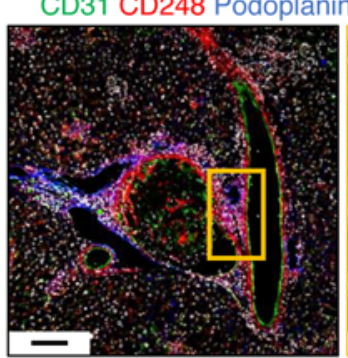

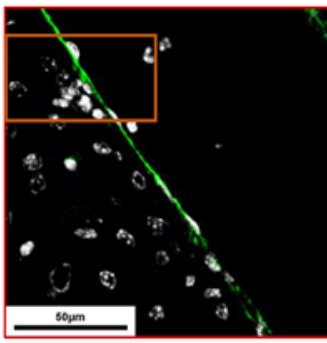
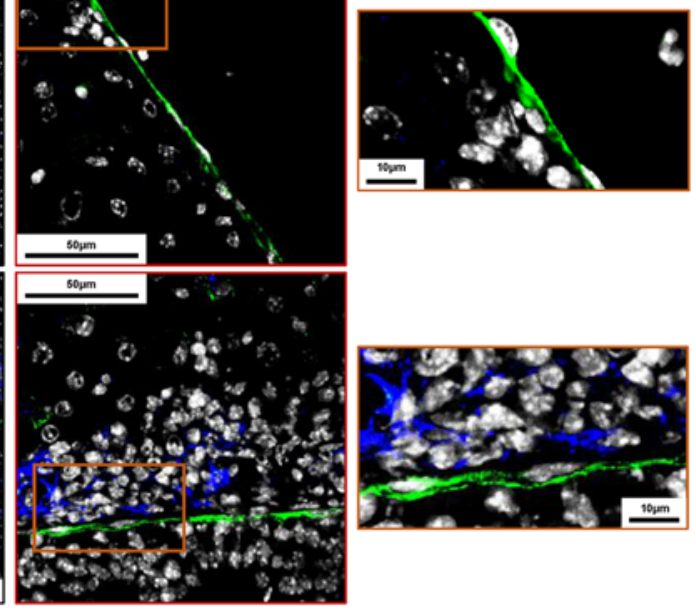

Figure 6. Podoplanin expression is elevated in the liver during infection. WT mice were infected i.p. as above for 7 days, and (A) podoplanin expression (green) was assessed in the liver alongside CD41 (red) and Hoechst (gray). (B) Podoplanin expression (blue) was examined with CD31 expression (green) in noninfected (top panel) and day 7-infected (bottom panel) sections; boxed areas are enlarged to the right-hand side. (C) Podoplanin (blue) expression by leukocytes was examined alongside CD41 (green), CD45.2 (red), and Hoechst (gray). (D) Podoplanin expression (blue) by stromal populations was examined alongside CD31 (green), CD248 (red), and Hoechst (gray). All images were obtained from frozen liver sections by fluorescent confocal microscopy. Scale bars: $100 \mu \mathrm{m}$ unless indicated otherwise. LV, lymphatic vessel. Images are representative of $\geq 3$ experiments with $\geq 4$ mice in each group.

did not express podoplanin before or during infection (Figure 6B). At day 7, podoplanin was highly expressed throughout the liver on leukocyte and nonleukocyte (CD45-) populations both within inflammatory foci and by cells that lie adjacent to the vascular endothelium (Figure 6C). These subendothelial podoplanin ${ }^{+}$cells included $\mathrm{CD} 248^{+}$cells, indicating they could include pericytes or fibroblasts among other stromal populations, although not all CD248 ${ }^{+}$cells express podoplanin (Figure 6D).

Podoplanin is expressed by multiple macrophage populations in the liver during infection. Podoplanin expression has been previously reported on macrophages during inflammation, and so $\mathrm{F} 4 / 80^{+}$ cells were further examined (24). Podoplanin is expressed by $\mathrm{F} 4 / 80^{+}$cells before and after infection (Figure 7A and Supplemental Figure $1 \mathrm{~A}) . \mathrm{F} 4 / 80^{+}$cells isolated from the liver were categorized according to expression of Ly6G, CD11c, CD11b, and Ly6C, and podoplanin expression of each population was measured by flow cytometry (Figure 7B and Supplemental Figure 2, A-C).

While the number of cells in most $\mathrm{F} 4 / 80^{+}$populations examined increased within 24 hours of infection (Figure 7C), the proportion of cells expressing podoplanin was low, typically around $5 \%$ (Figure 7D). By day 7 of infection, there was an increase in the numbers of cells in each of the 8 populations assessed (Figure
7C) and the proportion of cells expressing podoplanin increased, most notably in the $\mathrm{F} 4 / 80^{+} \mathrm{Ly} 6 \mathrm{G}^{\mathrm{lo}} \mathrm{CD} 11 \mathrm{c}^{+} \mathrm{CD} 11 \mathrm{~b}^{\text {hi }} \mathrm{Ly} 6 \mathrm{C}^{\mathrm{lo}}$ population (population 2; Figure 7, C-D). Thus, podoplanin expression by $\mathrm{F} 4 / 80^{+}$subsets was widespread and heterogeneous by the time thrombosis was established in the liver (Figure 7E).

Increased numbers of podoplanin-expressing cells after infection is dependent on IFN- $\gamma$ and TLR4. To determine whether there is a relationship between podoplanin expression and IFN- $\gamma$, we assessed IFN- $\gamma$ expression by the podoplanin ${ }^{+}$populations identified in Figure 7 using eYFP reporter mice (Figure 8A; representative FACS plots are shown in Supplemental Figure 3). Absolute numbers at all time points examined are shown in Supplemental Figure 4, A and B. At day 7 after infection, IFN- $\gamma$ expression was found in $\mathrm{F} 4 / 80^{+}$cells that were $\mathrm{CD} 11 \mathrm{c}^{+}$and $\mathrm{CD} 11 \mathrm{c}^{-}$and particularly in those populations that were CD11b- (Figure 8B). Proportionally, cells that were podoplanin ${ }^{+}$were more likely to express IFN- $\gamma$ (Figure 8B). In addition to $\mathrm{F} 4 / 80^{+}$cells, NK cells and similar numbers of invariant NKT (iNKT) cells were also found to express IFN- $\gamma$ after infection (Figure 8C and data not shown). Nevertheless, thrombosis is not dependent upon NKT cells, as it was present to a similar extent in CD1d-deficient mice, which lack NKT cells (Figure 8D). 
A

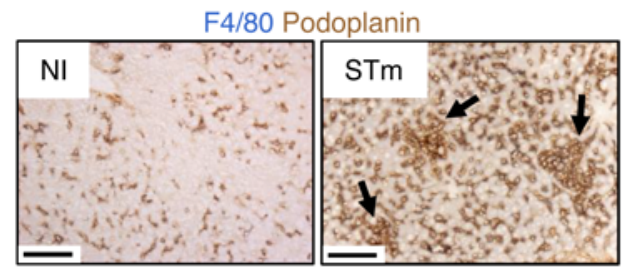

C

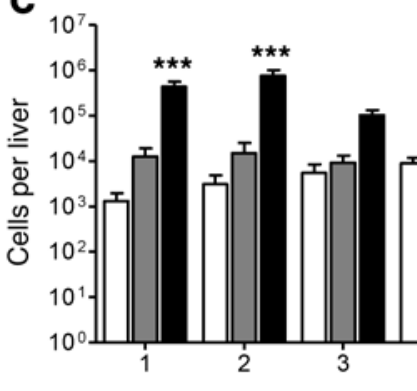

B

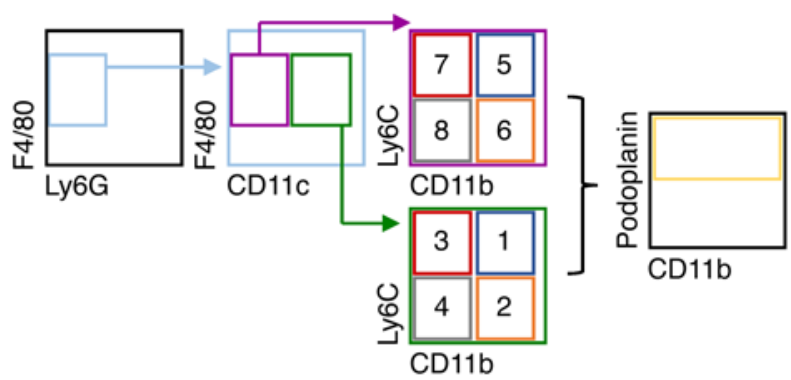

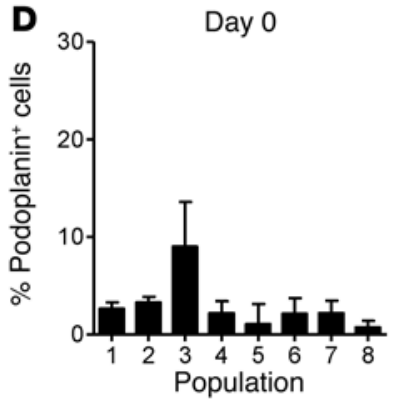

E

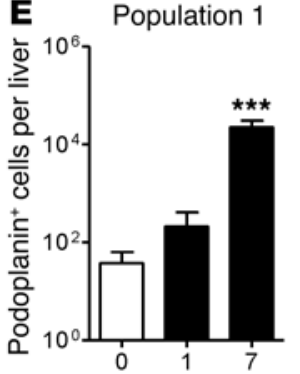

Time after infection (d)

Population 5

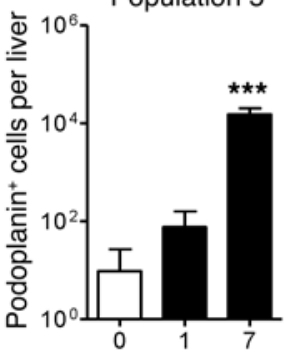

Time after infection (d)

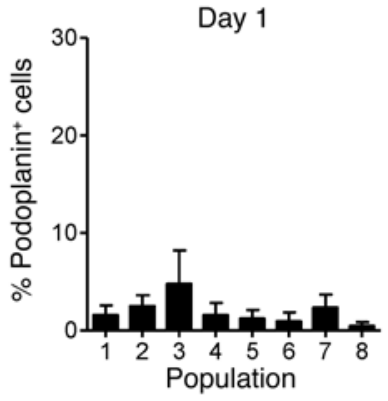

Population 2

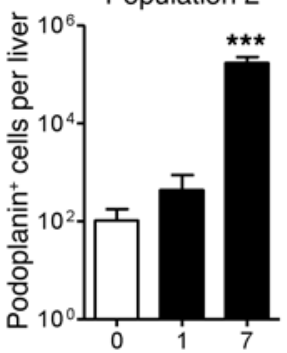

Time after infection (d)

Population 6

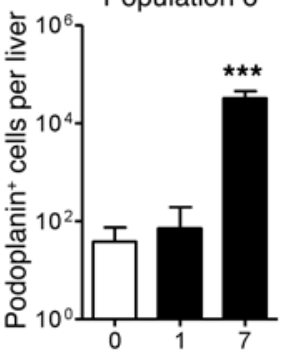

Time after infection (d)

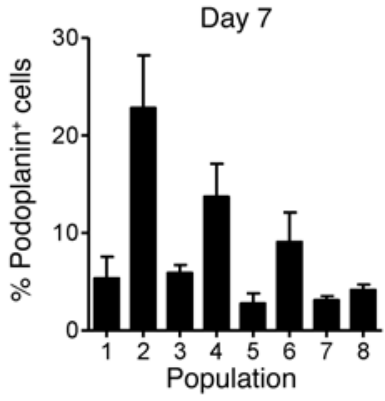

Population 3

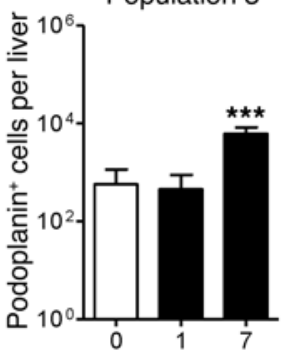

Time after infection (d)

Population 7

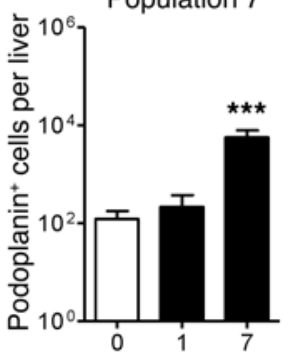

Time after infection (d)

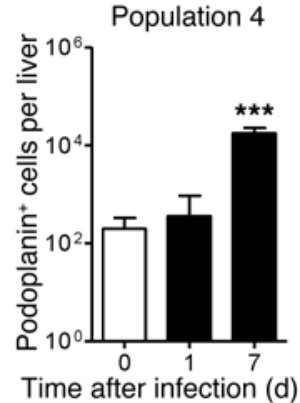

Population 8

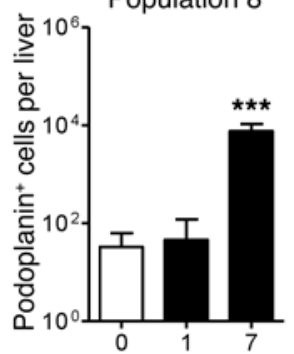

Time after infection (d)

Figure 7. Podoplanin is expressed by multiple macrophage populations in the liver during infection. WT mice were infected i.p. as above for 7 days, and (A) podoplanin expression (brown) was examined on frozen liver sections with F4/80 (blue). Scale bars: $100 \mu \mathrm{m}$. Arrows indicate inflammatory lesions. Podoplanin expression by macrophage populations was measured by flow cytometry on leukocytes isolated from livers. (B) The gating strategy used

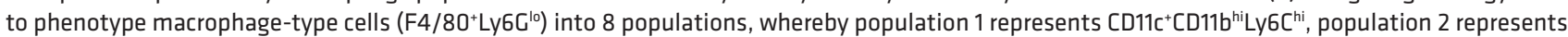

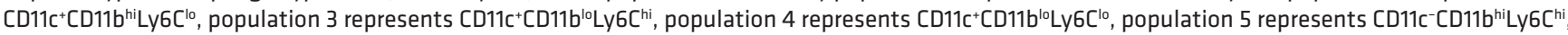

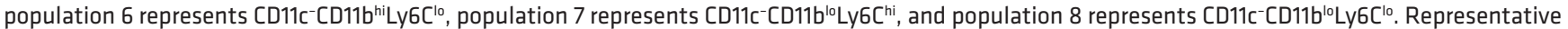
FACS plots are shown in Supplemental Figure 2. (C) Absolute numbers of cells in each population. (D) Proportion and (E) absolute numbers of podoplanin ${ }^{+}$ cells in each population. Graphs show mean + SD from a representative experiment (1 of 4 repeat experiments), with each group containing $\geq 4$ mice. Statistical significance was determined relative to noninfected mice. ${ }^{*} P \leq 0.05 ;{ }^{* *} P \leq 0.001$, 2-way ANOVA with Bonferroni's post-test (C) or 1-way ANOVA with Dunnett's test $(\mathbf{E})$. 
A
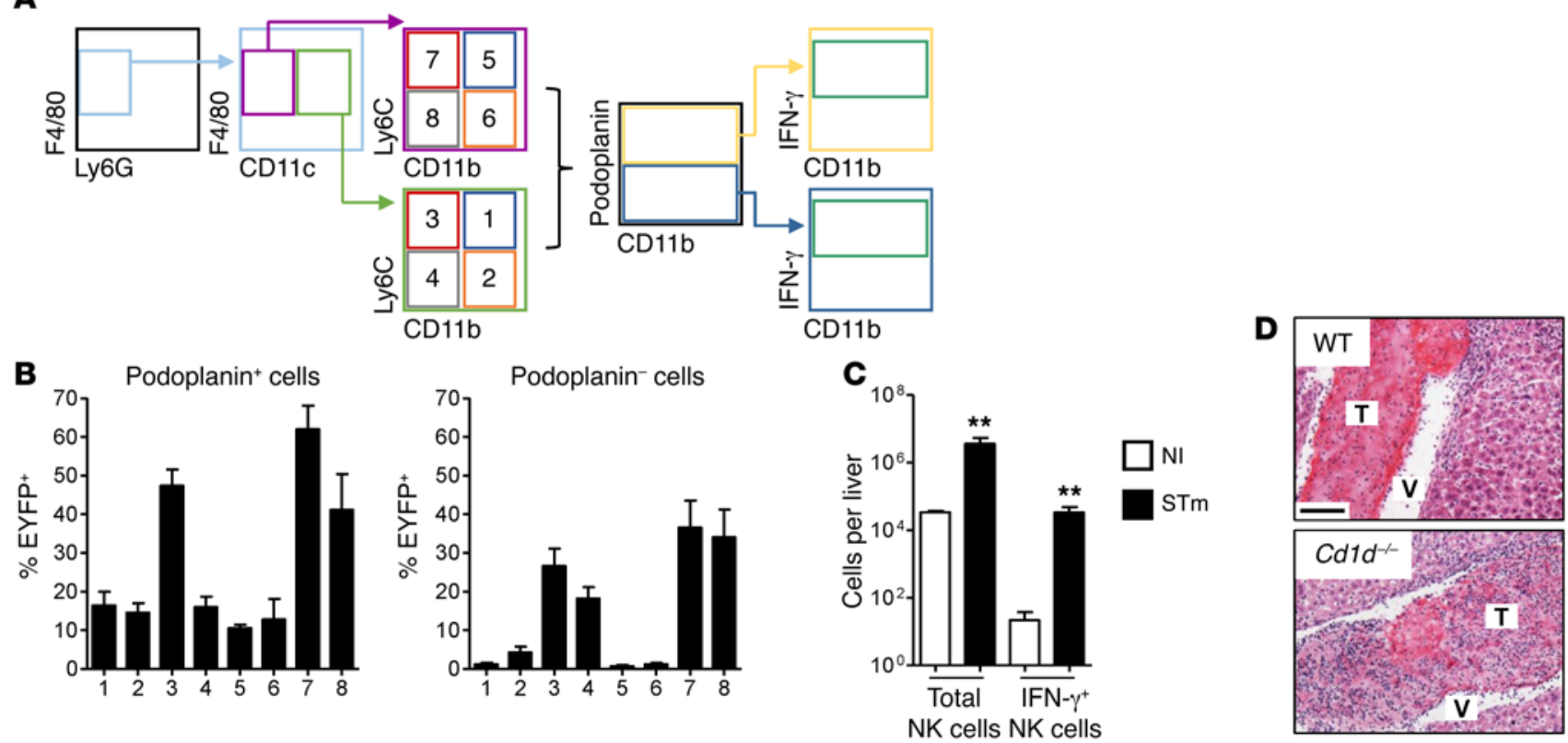

E

$\mathbf{F}$
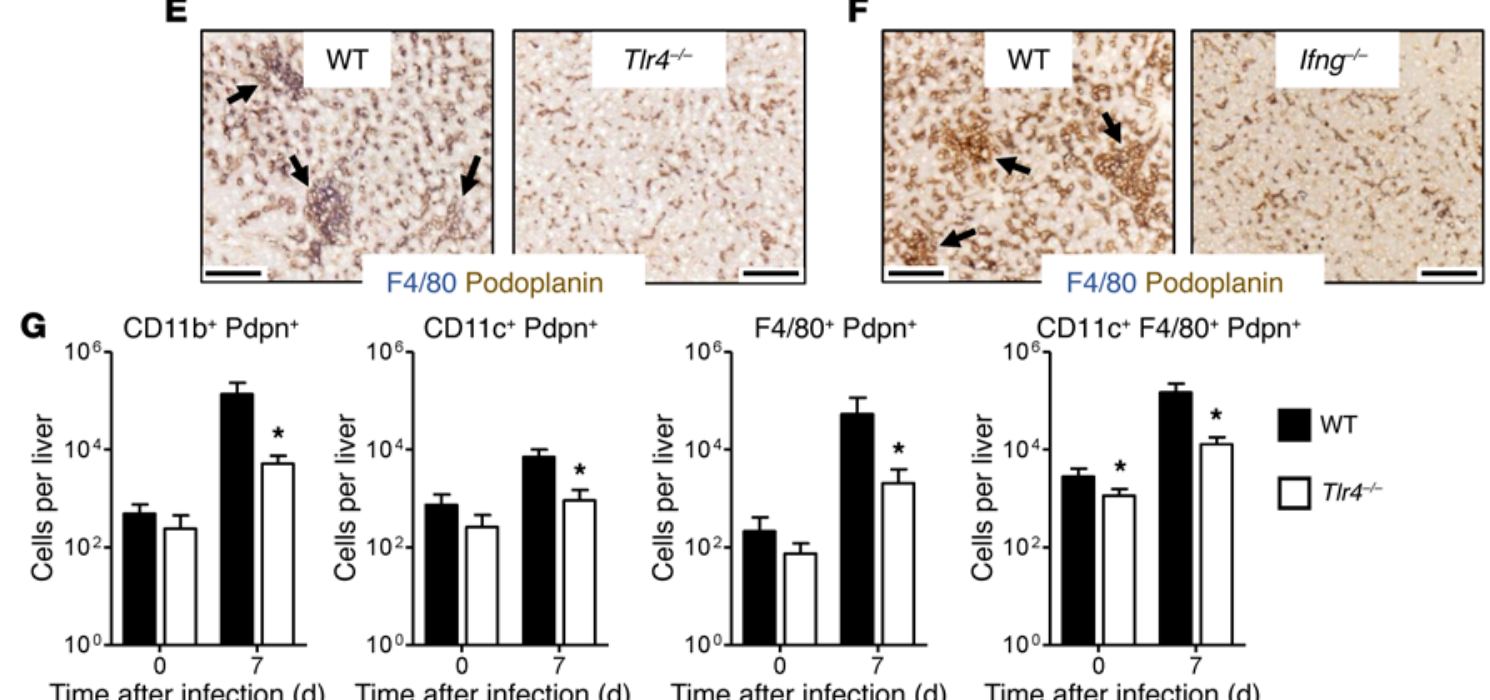

Time after infection

(d) Time after infection (d)

Time after infection (d)

Time after infection (d)

Figure 8. Increased numbers of podoplanin-expressing cells after infection are dependent on IFN- $\gamma$ and TLR4. EYFP IFN- $\gamma$ reporter mice were infected i.p. as above for 7 days, and IFN- $\gamma$ production was examined by flow cytometry in leukocytes isolated from the liver. (A) Macrophage populations were classified according to the gating strategy outlined in Figure 7. IFN- $\gamma$-producing cells were identified by EYFP expression. Representative FACS plots are shown in Supplemental Figure 3. (B) The proportion (at day 7 after infection) of podoplanin+ (left panel) and podoplanin- (right panel) cells in populations 1-8 that are EYFP+. (C) Number of NK cells (CD3- NK1.1 $1^{+}$) and IFN- $\gamma^{+}$NK cells per liver in noninfected and day 7-infected mice. (D) Cd1 $d^{-/-}$mice were infected as above, and thrombus development in the liver was examined by H\&E at day 7. (E) T/r4 ${ }^{-/-}$and (F) Ifng ${ }^{-/-}$mice were infected as above for 7 days, and podoplanin expression (brown) was examined by IHC with F4/80 expression (blue). (G) Leukocytes were isolated from livers of WT and TIr4 ${ }^{-/-}$mice, and podoplanin expression was measured by flow cytometry. Absolute numbers of podoplanin ${ }^{+}$cells from noninfected and day 7-infected mice. All experiments were performed twice with $\geq 4$ mice in each group at each time point. Data are shown as mean + SD. Statistical significance was determined relative to noninfected mice (C) or to WT mice (C). ${ }^{*} P \leq 0.05 ;{ }^{* *} P \leq 0.01$, Mann-Whitney sum of ranks test. All images are representative and are from frozen liver sections. Scale bars: $100 \mu \mathrm{m}$. Arrows indicate inflammatory lesions.

At day 7 after infection, the extent of podoplanin expression in IFN- $\gamma$ - or TLR4-deficient livers was similar to that seen in noninfected mice; numbers of podoplanin-expressing cells were reduced relative to those in infected WT mice (Figure 8, E-G). Therefore, TLR4 ligation and IFN- $\gamma$ production were required for podoplanin upregulation in addition to inflammatory foci development and thrombosis. Considering that the temporal and spatial upregulation of podoplanin is consistent with a potential role in inflammation and thrombosis and that the increase in its expression is absent in circumstances where thrombosis is also absent, we hypothesized that podoplanin upregulation provides the crucial link between inflammation and thrombosis.

CLEC-2 expression on platelets is required for thrombus development during infection. The interplay between podoplanin and CLEC-2 has been previously described during inflammation and has been shown to influence dendritic cell motility and the 
A
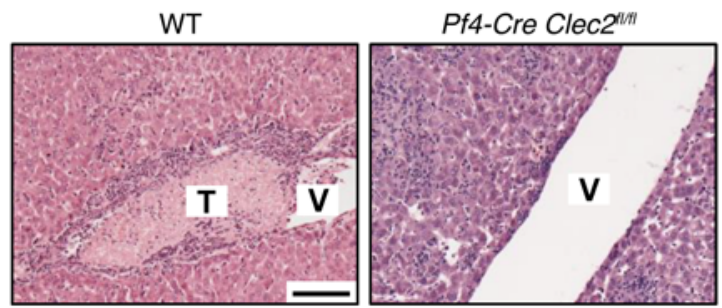

D

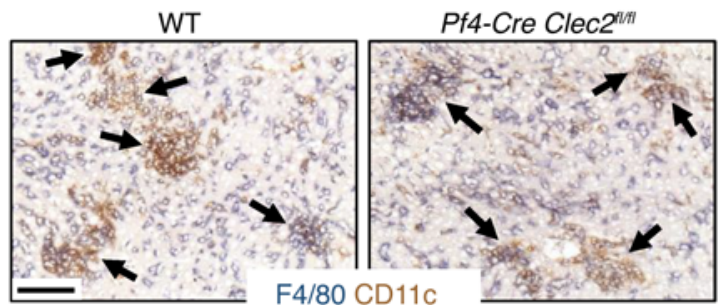

H

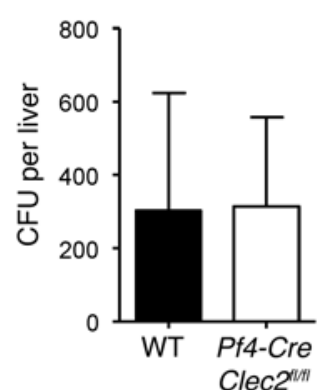

J

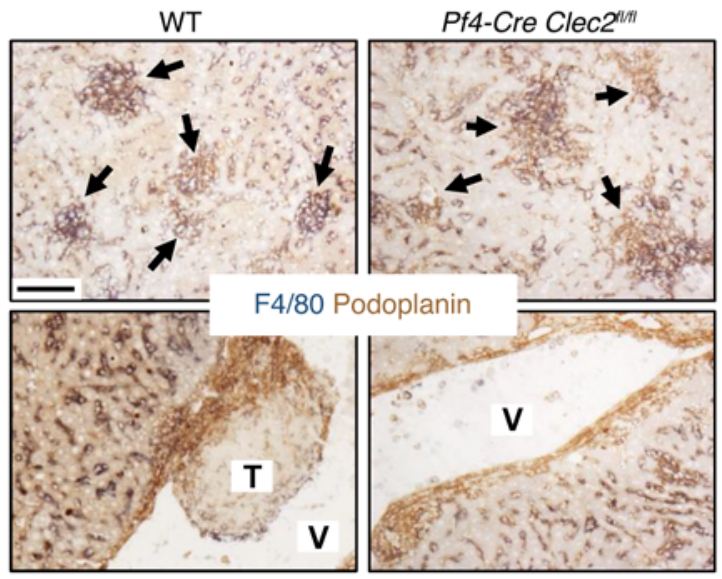

B

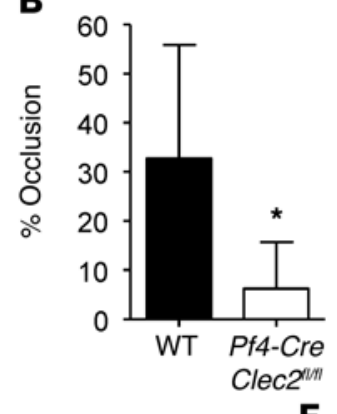

E

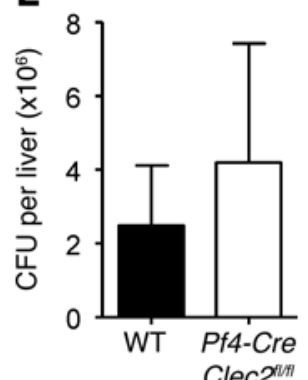

$\mathbf{F}$

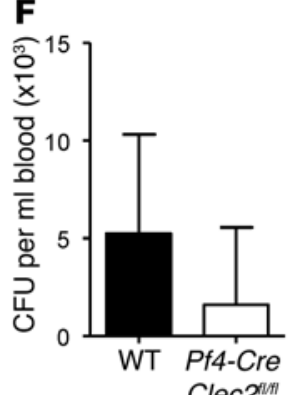

$\mathrm{CD}_{11 \mathrm{C}^{-}} \mathrm{F} 4 / 80^{+}$

K $\begin{aligned} \mathrm{CD} 11 \mathrm{c}^{+} \mathrm{F} 4 / 80 \\ \text { Podoplanin }\end{aligned}$

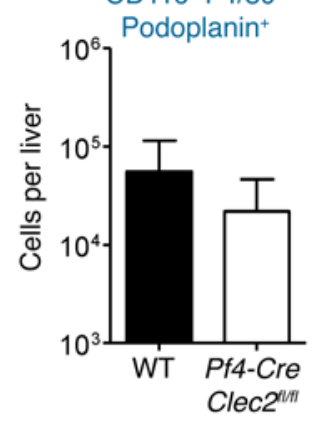

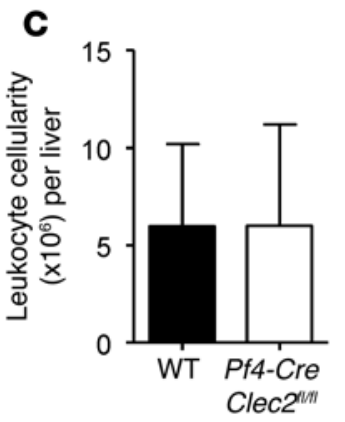

G

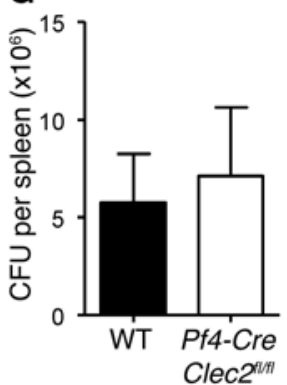

$\mathrm{CD}_{11 \mathrm{C}^{+}} \mathrm{F} 4 / 80^{+}$

Podoplanin +

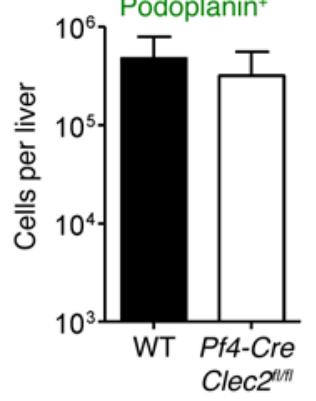

$\mathbf{L}$

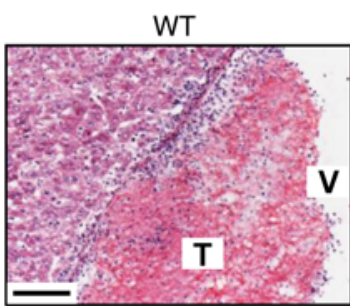

Gpvi $^{-}$Pf4-Cre Clec2 $2^{\text {III }}$
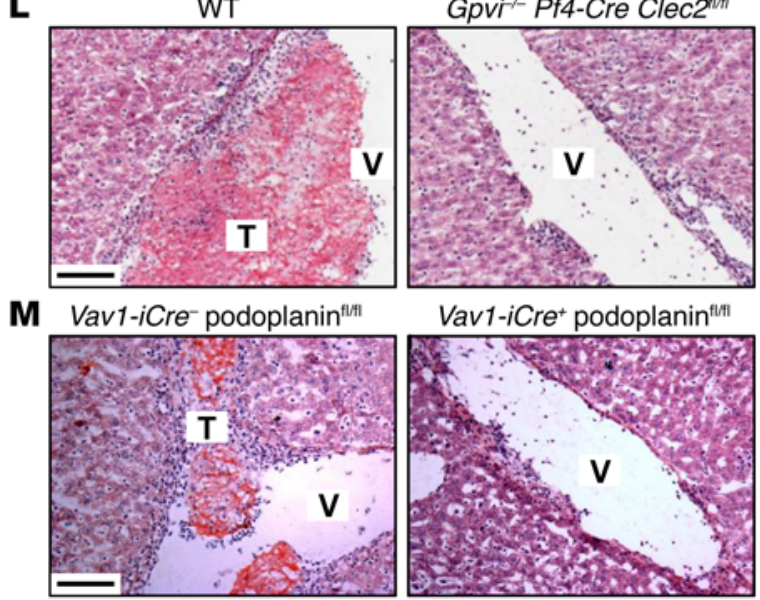

Vav1-iCre+ podoplanin ${ }^{\text {ftht }}$

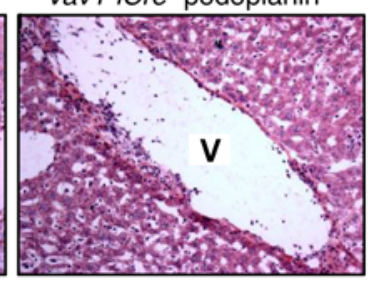

Figure 9. Thrombosis is dependent upon CLEC-2 expression on platelets. WT and Pf4-Cre Clec $2^{f / f l}$ mice (lacking CLEC-2 expression on platelets) were infected i.p. as above for 7 days. (A) Thrombosis was examined on paraffin-embedded liver sections using H\&E, and (B) vascular occlusion was quantified by point counting of large vessels per tissue section. (C) Absolute number of leukocytes isolated from the liver at day 7. (D) Leukocyte infiltration was examined by IHC: CD11c (brown), F4/80 (blue). Bacterial colonization of (E) liver, (F) blood, and (G) spleen at day 7. WT and Pf4-Cre Clec $2^{f / f f}$ mice were infected as above for 35 days, and bacterial colonization of (H) the liver and (I) spleen were enumerated. (J) Podoplanin expression (brown) was examined in parenchymal (top panels) and vascular (bottom panels) regions of frozen liver sections by IHC with F4/80 (blue) at day 7 after infection. Leukocytes were isolated from the liver at day 7, and podoplanin expression was measured by flow cytometry. Leukocytes were classified by expression of F4/80 and CD11c, as shown in Supplemental Figure 5. (K) Absolute numbers of podoplanin ${ }^{+}$leukocyte populations. (L) Gpvi ${ }^{\text {/- }}$ Pf4-Cre Clec $2^{f / f l}$ mice (lacking both CLEC-2 expression on platelets and constitutive GPVI expression) and (M) Vav1-iCre $e^{+}$podoplanin ${ }^{\mathrm{fl} / \mathrm{fl}}$ mice (lacking podoplanin expression on hematopoietic cells) and relevant littermate controls were infected as above, and thrombus development was examined on frozen liver sections by H\&E at day 7 after infection. Data in $\mathbf{L}$ and $\mathbf{M}$ are each representative of 2 experiments where $n \geq 4$ mice per group; remaining data and images are representative of experiments performed at least 4 times using groups containing $\geq 4$ mice. Graphs show mean + SD. Statistical significance was determined relative to WT mice. ${ }^{*} P \leq 0.05$, Mann-Whitney sum of ranks test. Scale bars: $100 \mu \mathrm{m}$. Arrows indicate inflammatory lesions. 
A

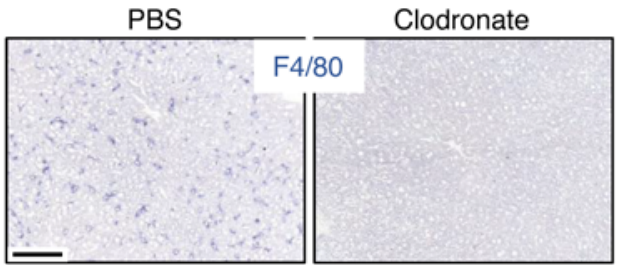

C

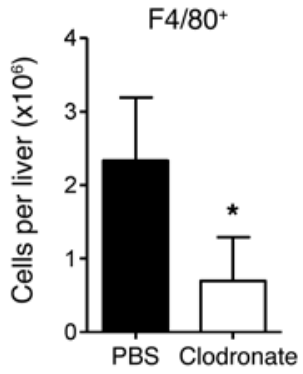

E
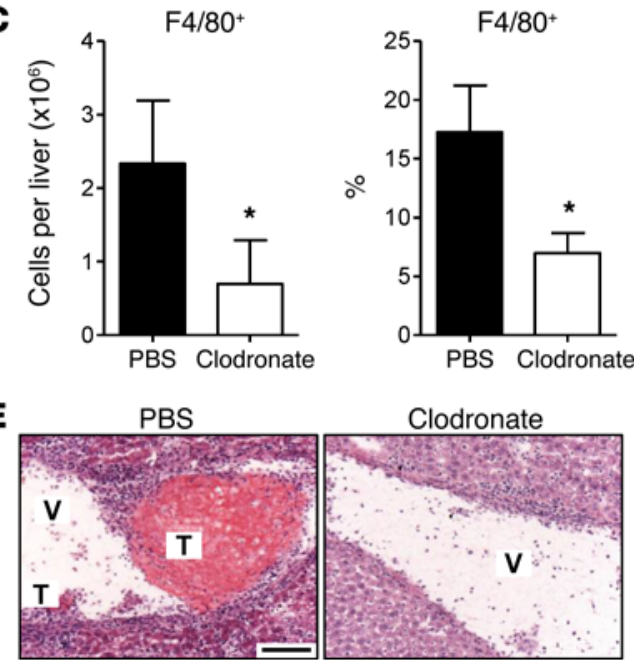

B

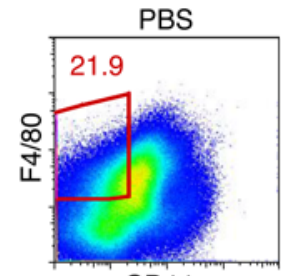

CD11c

D

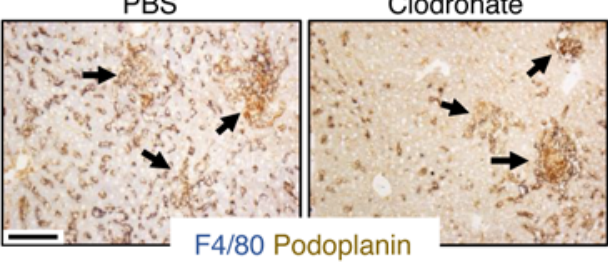

F

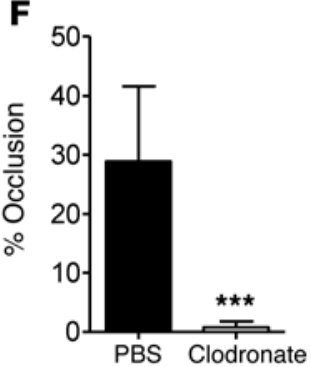

Figure 10. Clodronate-sensitive cells mediate thrombus development. (A) WT mice were treated i.p. with PBS-loaded or clodronate-loaded liposomes for 24 hours, and macrophage depletion was examined on frozen liver sections by IHC: F4/80 (blue). Images are representative of an experiment with 3 mice per group. (B-F) WT mice were treated with PBS- or clodronate-loaded liposomes 24 hours prior to infection with $5 \times 10^{5}$ CFU S. Typhimurium and every 48 hours subsequently. Leukocytes were isolated from the liver at day 7 after infection, and macrophage populations were examined by flow cytometry. (B) Representative FACS plots and (C) absolute number/proportion of F4/80+ cells per liver at day 7 after infection. (D) Macrophage expression of podoplanin (brown) was examined by IHC in conjunction with F4/80 (blue). (E) Thrombosis was examined by H\&E staining and (F) was quantified by point counting of large vessels per tissue section. Data in B-F are representative of 2 experiments where $n=5$ in each group. Graphs show mean + SD; statistical significance was determined by comparison between PBS liposome- and clodronate liposome-treated mice. ${ }^{*} P \leq 0.05 ;{ }^{* * *} P \leq 0.001$, Mann-Whitney sum of ranks test. All images were obtained from frozen liver sections. Scale bars: $100 \mu \mathrm{m}$. Arrows indicate inflammatory foci.

maintenance of vascular integrity under thrombocytopenia (32, 33). While platelet-expressed CLEC-2 has been implicated in the maintenance of vascular integrity at sites of inflammation, this role does not require platelet aggregation and thrombus formation (33). To investigate a role for CLEC-2 in $S$. Typhimurium-induced thrombosis, we studied $\mathrm{Clec} 2^{f / f l}$ mice with a platelet-specific deletion of CLEC-2 achieved using expression of Cre under a platelet factor 4 promoter (Pf4-Cre Clec $2^{f l f l}$ ). In these mice, thrombosis was markedly reduced at day 7 after infection, with most areas of the liver vasculature devoid of thrombus formation in most mice, demonstrating a critical role for platelet CLEC-2 (Figure 9, A and B). Importantly, this diminished thrombosis was not due to a lower level of inflammation or bacteremia, as leukocyte infiltration, inflammatory lesion development, and bacterial numbers were similar to those of infected littermate controls, indicating that other features of the infection were conserved despite the lack of CLEC-2 on platelets (Figure 9, C-E, and ref. 34). Similarly, both bacteremia and bacterial burden of other peripheral tissues such as the spleen were comparable to those of littermate controls, further emphasizing the maintained control of infection in the absence of CLEC-2-activated platelets (Figure 9, F and G). Moreover, $\mathrm{Pf} 4$-Cre Clec $2^{f / f l}$ mice were able to clear bacteria from the liver and spleen with kinetics similar to those of littermate controls by day 35 after infection (Figure $9, \mathrm{H}$ and I), indicating that thrombosis does not impede infection resolution in mice where inflammation is maintained. In addition, there was no feedback from the loss of CLEC- 2 on podoplanin expression in the liver, indicating that the loss of thrombi is directly related to the inability of podoplanin-expressing cells to interact with platelets (Figure 9, J and K, and Supplemental Figure 5, A and B). Since occasional thrombi were seen in the Pf4-Cre Clec $2^{f / f l}$ mice, it is possible that these could be mediated by GPVI. However, this was not the case, as residual thrombi were also present in infected $\mathrm{Pf} 4$-Cre Clec $2^{f / f l}$ mice that were also deficient in GPVI (Figure 9L). Finally, mice with reduced podoplanin expression $\left(\mathrm{Vav1}-i \mathrm{Cre}^{+}\right.$podoplanin $^{\mathrm{f} / \mathrm{fl}}$ mice) also had impaired thrombosis compared with Vav1-iCrepodoplanin $^{\mathrm{fl} / \mathrm{fl}}$ littermate controls (Figure 9M). Therefore, during $S$. Typhimurium infection, the podoplanin-CLEC-2 dyad controls thrombosis induction in a manner independent of GPVI.

Clodronate-sensitive cells mediate thrombus development. Since ligation of CLEC-2 on platelets is required for thrombus induction and $\mathrm{F} 4 / 80^{+}$cell populations constitute a substantial source of podoplanin, it is possible that the lack of thrombosis in infected $\mathrm{Tlr}^{-/-}$and Ifng $\mathrm{g}^{-/}$mice is due to insufficient podoplanin-expressing macrophage numbers in the liver. To test whether monocyte-lineage cells were therefore sufficient to drive platelet activation and thrombus formation, mice were treated before and during infection with clodronate liposomes 
A

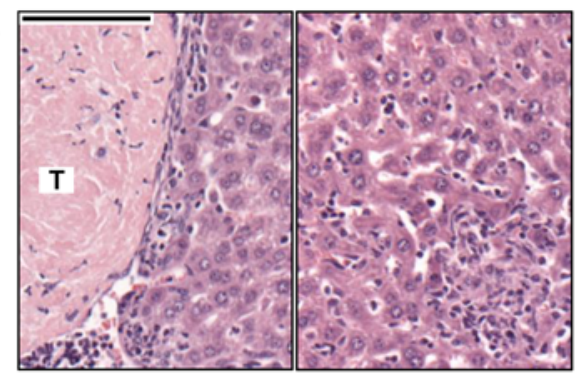

C

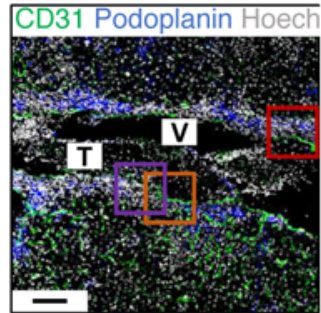

D
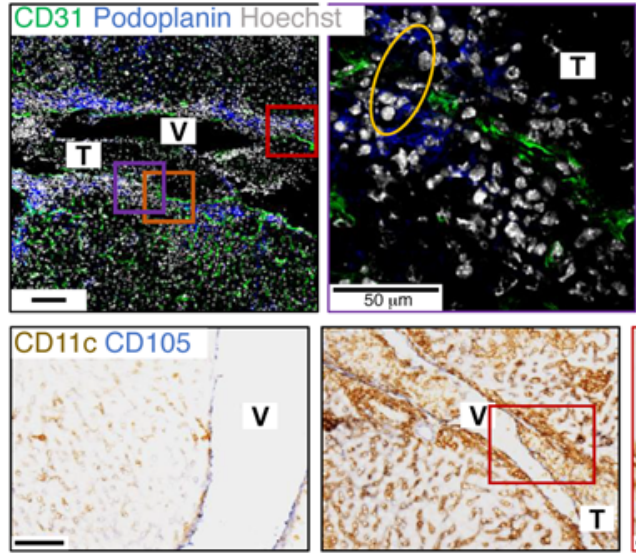
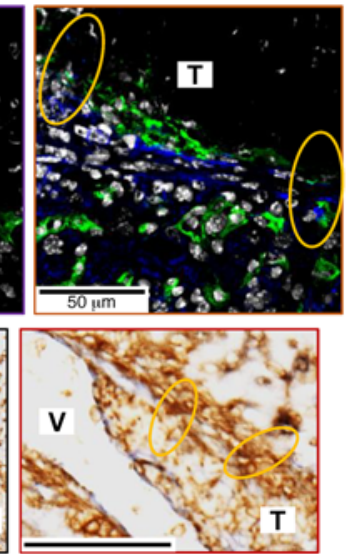

$\mathbf{F}$
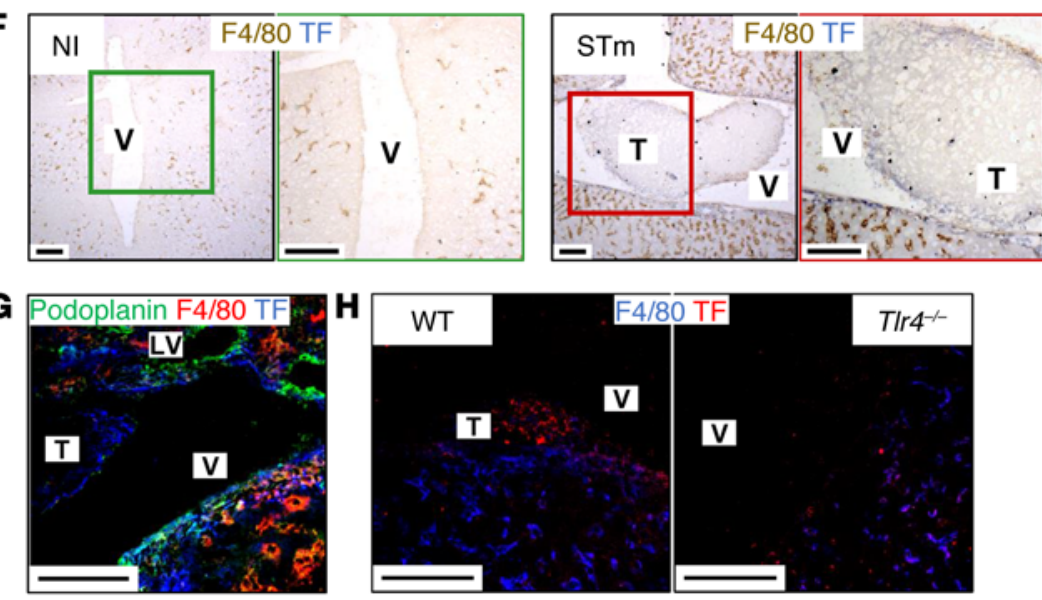

I
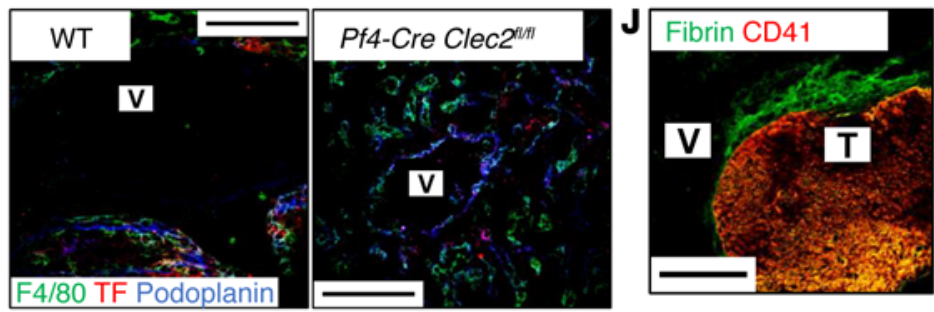

Figure 11. Thrombi are anchored at sites of endothelial perturbation. WT mice were infected i.p. as above for 7 days. (A) Paraffin-embedded liver sections were examined at high magnification by $\mathrm{H} \& \mathrm{E}$ in regions adjacent to the portal vein (left panel) and within the parenchyma (right panel). (B-J) Frozen liver sections were examined by IHC/fluorescent confocal microscopy staining for (B) CD41 (blue), (C) CD31 (green), podoplanin (blue), and Hoechst (gray). Boxed regions are shown at a higher magnification in second, third, and fourth panels. (D) CD11c (brown), CD105 (blue). Left panel shows noninfected, middle panel shows day 7 infected, and right panel shows higher magnification of boxed region. (E) CD31 (green), TF (using antibody 1H1) (red), podoplanin (blue). Top panel shows noninfected, and bottom panel shows day 7 infected. (F) F4/80 (brown), TF (blue; using antibody AF3178). Left panel shows noninfected, and right panel shows day 7 infected; right image is an enlargement of the boxed region. (G) Podoplanin (green), F4/80 (red), and TF (blue; using antibody AF3178) (day 7 infected). (H) WT and TIr4 ${ }^{-/-}$mice and (I) WT and Pf4-Cre Clec $2^{f / f I}$ mice were infected as above for 7 days, and liver sections were stained for (H) F4/80 (blue), TF (red; using antibody 1H1), (I) F4/80 (green), TF (red; using antibody 1H1), podoplanin (blue), and (J) fibrin (green), CD41 (red) (WT, day 7 infected). Images in A-G, I, and J are representative of a minimum of 3 experiments, where $n \geq 4$ mice per group. Images in $\mathbf{H}$ are representative of 2 experiments with $\geq 4$ mice per group. Scale bars: $100 \mu \mathrm{m}$, unless indicated. Yellow circles indicate areas of compromised endothelial integrity. 
or PBS liposomes. Clodronate treatment prior to infection reduced $\mathrm{F} 4 / 80^{+}$cell numbers in the liver (Figure 10A). After infection, some increase in $\mathrm{F} 4 / 80^{+}$cells was observed; however, $\mathrm{F} 4 / 80^{+}$cell numbers and proportions were significantly lower at day 7 after infection relative to PBS liposome treatment (Figure $10, \mathrm{~B}$ and $\mathrm{C}$ ). Reduced $\mathrm{F} 4 / 80^{+}$cell numbers in the liver were paralleled by reduced podoplanin expression on tissue sections after infection and by the abrogation of thrombus development (Figure 10, D-F). Therefore, clodronate-sensitive monocytelineage cells regulate the induction of thrombosis through a podoplanin-CLEC-2-dependent mechanism.

Endothelial damage exposes podoplanin, facilitating CLEC-2dependent platelet activation. Having demonstrated that platelet activation in this model is CLEC-2 dependent and that podoplanin expression by macrophage subsets is required to facilitate this activation, we wanted to identify where this platelet-podoplanin interaction occurs. Considering podoplanin is expressed by Kupffer cells (among other macrophage subsets) that are primarily localized to the sinusoids, it seemed plausible that platelet activation may occur within the parenchyma. However, thrombi were not detected within the sinusoids, either in the presence or absence of infection, suggesting that platelet exposure to Kupffer cells during normal perfusion of the liver is not sufficient for thrombus induction (Figure 11A and data not shown). Instead, we saw regions adjacent to the vascular endothelium of the portal vein where platelets appeared to "anchor" the thrombi to the side of the vessel wall (Figure 11B). In these regions, the platelets formed miniprotrusions into the parenchyma, suggesting platelets become activated at points where there is perturbation of the integrity of the portal vein endothelium. At such points, there were interruptions in the $\mathrm{CD} 1^{+}$vascular endothelium (Figure $11 \mathrm{C}$ ). This discontinuous endothelial staining was also observed when other markers, such as CD105, were used and suggests this is not simply reflecting some change in CD31 expression (Figure 11D).

Expression of tissue factor (TF), a key component of coagulation, by macrophages has been previously shown to be enhanced in response to TLR4 stimulation by LPS (35). While TF is not readily detected in noninfected mice, at day 7 after infection, TF staining could be seen in perivascular sites, although it was not expressed by CD $31^{+}$vascular endothelium (Figure 11, E-G). After infection, $\mathrm{TF}$ was detected on $\mathrm{F} 4 / 80^{+}$and $\mathrm{F} 4 / 80^{-}$cells; however, only a small proportion of $\mathrm{F} 4 / 80^{+}$cells expressed $\mathrm{TF}$ (Figure $11 \mathrm{~F})$. Occasionally, TF was observed at the same anatomical sites as podoplanin and could sometimes be found on the same cells; however, TF was not expressed by podoplanin ${ }^{+}$lymphatic endothelial cells (Figure 11, E and G). TF staining could be associated with thrombi themselves, although this was not observed on all thrombi and the staining was localized to the periphery of thrombi (Figure 11, F and G). An absence of thrombosis in TLR4deficient/macrophage-depleted animals could also reflect a reduction in $\mathrm{TF}$ present. In $\mathrm{Tlr} 4^{-/-}$mice, $\mathrm{TF}$ expression was reduced, but still present and detectable on $\mathrm{F} 4 / 80^{+}$cells (Figure $11 \mathrm{H}$ ). This suggests that the absence of thrombosis in TLR4deficient mice is unlikely to be due to an absence of TF. Similarly, $\mathrm{TF}$ was detected on $\mathrm{F} 4 / 80^{+}$cells in Pf4-Cre Clec $2^{f / f l}$ mice at day 7 after infection, further suggesting that $\mathrm{TF}$ expression is not sufficient for thrombus development (Figure 11I).
The identification of $\mathrm{TF}$ within thrombi suggests that the coagulation cascade may also contribute to the propagation of this pathway of thrombosis. Indeed, $\mathrm{CD} 41^{+}$platelet thrombi costained with anti-fibrin, indicating that thrombi were rich in fibrin, the end product of the coagulation cascade (Figure 11J). In addition, thrombi also stained positive for $\mathrm{VWF}$, as did CD31+ vascular endothelial cells (Supplemental Figure 6). This suggests that, although the induction of the thrombotic pathway is through CLEC-2 activation of platelets, the downstream processes share similarities with classical pathways of thrombosis.

\section{Discussion}

This study reports a mechanism that links infection, inflammation, and thrombosis. Based on our findings, we propose a model as to how systemic $S$. Typhimurium infection induces thrombosis. After infection, there is a differential capacity of the bacterium to colonize solid organs, such as the liver, or to persist in the blood. The bacteria in the liver cause a TLR4- and IFN- $\gamma$-dependent accumulation of podoplanin-expressing monocyte-lineage cells. As a consequence of the combination of bacteremia, infection of the liver, inflammation, and cellular migration into the liver, the vascular endothelium becomes damaged, exposing platelets to these podoplanin-expressing cells (both of monocytic lineage and podoplanin ${ }^{+}$cells of other CD $45^{-}$lineages). This combination of podoplanin sources results in platelet activation via CLEC-2. In this process, infection is the triggering event for the development of inflammation that leads to the thrombotic response, with the maintenance of the response being independent of continuing infection and instead paralleling the regulation of inflammation. Nevertheless, it is unclear whether an individual thrombus persists throughout the infection or whether there were cycles of thrombus formation and collapse. Elucidating this will be highly relevant for understanding how to intervene in this process.

While the importance of macrophages and IFN- $\gamma$ in the immune response to $S$. Typhimurium is well established, particularly with regard to bacterial clearance $(36,37)$, this TLR4-dependent inflammatory cascade has not been previously associated with altered podoplanin expression. Similarly, while podoplanin expression by macrophages has been described during inflammation (24) and has been putatively associated with TLR4 signaling in vitro (38), here, we provide in vivo evidence linking podoplanin expression to TLR4/IFN- $\gamma$-dependent inflammation. Furthermore, we demonstrate a functional application for podoplanin upregulation in CLEC-2-dependent thrombosis. Considering the increased numbers of CD248 ${ }^{+}$cells previously shown in the spleen following Salmonella infection (39), here, we demonstrate by clodronate depletion that podoplanin expression by nonmacrophage populations is insufficient for thrombus development. Further investigation will be required to determine whether $\mathrm{TF}$, which is also seen, actively contributes to this process.

The benefits of current antiplatelet medications, such as heparin, to treat thrombosis associated with infection are unclear (40). Indeed, we have shown that $S$. Typhimurium-mediated thrombosis in the liver is not altered by pretreatment with the orally available antiplatelet drugs aspirin and clopidogrel, which inhibit thromboxane formation and block the platelet $\mathrm{P} 2 \mathrm{Y}_{12}$ ADP recep- 
tor, respectively (Figure 4). Moreover, seeing as liver injury during infection is relatively mild (Figure 2, F-J), it is unlikely to compromise the conversion of clopidogrel to its active metabolite.

The identification of CLEC-2 as a critical player in this pathway opens the possibility of the development of a new class of antithrombotic agent with minimal, if any, effect on hemostasis or risk of increased bleeding. An important consideration in this context is the maintained separation of lymphatic/blood vasculature in the adult, a process that has been argued to be reliant on the interaction between CLEC-2 and podoplanin (41). Therefore, the possible compromised integrity of de novo angiogenesis during infection must be accounted for if this pathway is to be manipulated therapeutically, although any such intervention would likely only be used for short periods. The only known endogenous ligand for CLEC-2 is podoplanin, which is absent in the vasculature and at sites of acute tissue injury. This is consistent with the observation that tail bleeding times in mice, the most widely used model of hemostasis, have been shown to be minimally altered in mice deficient in CLEC-2 (42-44). A role for CLEC-2 in thrombosis has been observed in some but not all studies, notably in association with GPVI (44).

A key point to examine is whether this mechanism of infection- and inflammation-driven thrombosis is beneficial to the host in that it may provide a pathway of bacterial capture and removal, as recently reviewed (14). However, we did not find evidence for a contribution of thrombosis to limiting bacteremia early during infection in our model, where thrombosis persisted for weeks after bacteremia was absent and tissue bacterial numbers were resolving. There are a number of potential reasons for this, some of which may relate to the model and some to the nature of the infection. Perhaps central to the latter point is the natural history of this infection in humans, which is characterized by high bacterial numbers in tissues such as the spleen, liver, and bone marrow, but low numbers in the blood (3). In a recent controlled study of typhoid infection in human volunteers, bacteremia occurred in the majority of cases, but subjects had less than 2 bacteria per $\mathrm{ml}$ of blood (45). Such a modest bacteremia is also a feature of invasive nontyphoidal Salmonella infections. Even in humans who have additional complications, such as HIV coinfection, bacterial densities were typically less than 10 bacteria per ml of blood (46), and yet it is culture-confirmed bacteremia that is a leading prognostic indicator for poor outcome.

This is a substantially different presentation from those of many more "classical" infections associated with thrombosis, such as pneumococcal or staphylococcal infections, where bacterial burdens can reach many magnitudes of order higher (47, 48). One interpretation of this is that there are multiple potential mechanisms that can be involved in inducing thrombosis after infection, some of which may be influenced by the predominant distribution of bacteria between the blood and other sites. The low bacteremia associated with invasive Salmonella infections influence the potential of thrombosis to be of benefit to the host. It is possible that thrombosis/platelet activation has additional effects on the host that are independent of controlling bacterial numbers, such as maintaining vessel integrity after damage. Platelets contain multiple immunomodulatory factors that could be released during thrombus generation (49). Nevertheless, thrombosis is likely to be common; one study found 3 of 5 patients who died from typhoid had evidence of disseminated intravascular coagulation $(50,51)$.

There are multiple potential scenarios as to how thrombosis may contribute to the outcome of infection. Thrombosis may help restrict bacterial dissemination by limiting bacterial trafficking through the blood. Alternatively, if thrombi exceed a certain size, they may become harmful. An exaggerated thrombotic response may result in the development of unstable thrombi; it is possible to envisage that such thrombi may break up (embolize), leading to additional complications, such as cerebral infarction or pulmonary embolism. Whether this occurs is likely to reflect a combination of factors that include the extent of thrombosis within an individual site and the stability of an individual thrombus. Central to understanding the role of thrombosis in infection will be understanding the spectrum of manifestations it can take in an individual host.

Our ability to interrogate this in depth using the genetically altered mice in this study is complicated by the nature of the murine Salmonella infection model, where susceptibility is strongly influenced by the genetic background of the mouse strain used (52). In hypersusceptible mice, such as those on a C57BL6/J or BALB/c background, use of an attenuated Salmonella strain enables the infection to be followed for many weeks. The compromise associated with using this model is that the extent of the infection is not as severe as that observed using resistant strains of mice with fully virulent strains of bacteria. Evidence from other descriptive studies shows that when mice are infected with more virulent strains of Salmonella, thrombi can form in other sites such as the spleen, and so thrombosis is not necessarily restricted to the liver (9). Further studies are needed to identify whether the mechanism for thrombosis identified here is applicable to other systemic infections such as those caused by Escherichia coli, which can cause hemolytic uremia syndrome in certain populations (53).

It is also noteworthy that, while inflammation and thrombosis were abolished in TLR4- and IFN- $\gamma$-deficient mice and in mice treated with clodronate, a residual level of thrombosis was seen in CLEC-2-deficient mice. A similar level of residual thrombus formation was seen in mice double deficient for CLEC-2 and the collagen receptor GPVI, indicating that this residual response is not mediated by this classical mechanism of platelet activation. This may suggest that additional minor mechanisms that were triggered by the TLR4-mediated events early after infection were also involved, but it may simply reflect CLEC-2 engagement being the final step in this cascade, and so some infrequent thrombosis could still be triggered.

In this model, thrombosis is mirrored by decreased circulating platelets; thus, it is plausible that this thrombocytopenia is simply a reflection of platelet consumption, a concept supported by the lack of thrombocytopenia in TLR4- and IFN- $\gamma$-deficient mice. However, considering that platelet numbers drop within 24 hours, several days before thrombi appear, it is probable that other mechanisms also contribute to thrombocytopenia. One possible way is via platelet development, which may be affected by the dramatic effect of this infection on bone marrow progenitor populations (54). Interestingly, thrombocytopenia is often observed after Salmonella infections in mice and humans and has been observed in humans infected with an attenuated strain of Salmonella given as a 
treatment against cancer $(9,55,56)$. Therefore, despite the effects that molecules such as TLR4 have on influencing platelet numbers after LPS challenge (57), the thrombocytopenia observed during infection is likely to be due to a combination of factors.

The present results may provide a mechanism to explain some of the deaths observed after invasive nontyphoidal Salmonella infections in infants in sub-Saharan Africa. In these cases, when infants present at clinic with bacteremia, up to $20 \%$ will die within 24 hours, despite the modest bacteremia associated with systemic nontyphoidal Salmonella infections (58-60). If so, it would suggest that understanding immunopathology after infection should be as important a focus of research for control of the sequelae of infection as trying to identify how to restrict bacterial numbers through antimicrobial treatment. For instance, in this study, we observed reduced cellular infiltration and negligible thrombus development in Ifng $\mathrm{g}^{-/}$mice, despite there being 10-fold higher bacterial numbers at day 7 .

The pathway that we describe here may also play a role in other conditions associated with inflammation and thrombosis, such as secondary hemophagocytic lymphohistiocytosis, a life-threatening condition associated with hyperinflammation due to dysregulated proliferation of lymphocytes and macrophages (61). If a role for podoplanin and CLEC-2 emerges in these clinical conditions, it will highlight this interaction as a key target for therapeutic intervention. Understanding when and how podoplanin and CLEC-2 are induced and interact will provide numerous new opportunities to therapeutically intervene without the risk of increased bleeding that is associated with other forms of antithrombotic therapy.

\section{Methods}

Mice. C57BL/6J mice and BALB/c mice (6 to 8 weeks) were obtained from Harlan OLAC Ltd. or from in-house colonies, respectively. Tlr $4^{-/}$ mice were from Oriental Bioservice. Ifng ${ }^{-/-}$mice, Rag1 $1^{-/-}$mice, IfngeYFP (GREAT) reporter mice, Vav1-iCre $e^{+}$podoplanin ${ }^{\mathrm{fl} / \mathrm{fl}}$ mice (obtained from Jackson Laboratories), and Pf4-Cre Clec $2^{f / f l}$ mice are described elsewhere (41, 62-65). Gpvi-/ mice (66) were a gift from Jerry Ware (University of Arkansas, Fayetteville, Arkansas, USA). Cd1 $d^{-/-}$mice were originally from Luc Van Kaer (Vanderbilt University Medical Centre, Nashville, Tennessee, USA) (67). Gpvi-/- Pf4-Cre Clec $2^{f / f l}$ mice were generated by breeding of $\mathrm{Gpvi}^{-/} \mathrm{Pf} 4-\mathrm{Cre} \mathrm{Clec}^{\mathrm{fl} / \mathrm{C}}$ with $\mathrm{Gpvi}^{-/}$ Clec $2^{f / f l}$ mice in-house at the Biomedical Services Unit (BMSU), University of Birmingham. All strains of genetically altered mice were on a C57BL/6J background with the exception of $T l r 4^{-/-}$mice, which were on a BALB/c background. Control mice were matched by genetic background, age, and sex. All mice were housed at the BMSU.

Bacteria and infection protocol. Mice were infected i.p. or i.v. with $5 \times$ $10^{5}$ attenuated Salmonella Typhimurium SL3261 as described previously (68). Noninfected or mock PBS-immunized mice were used as controls. Tissues were removed at the indicated time points after infection.

Clodronate treatment. Treatment with clodronate liposomes was performed as described elsewhere (69-72). Mice were treated i.p with $200 \mu \mathrm{l}$ clodronate liposomes or PBS liposomes 24 hours before $S$. Typhimurium infection (i.p.) and every 2 to 3 days subsequently.

Antiplatelet treatments. WT mice were infected as described above. Aspirin (30 mg/l, freshly prepared every other day) was administered daily via drinking water beginning on day 5 after infection, and treatment was maintained constantly until day 13. Clopidogrel
(Plavix) tablets $(75 \mathrm{mg}$ ) were ground to a fine powder and dissolved (as an emulsion) in $0.5 \%$ methylcellulose solution. Mice were treated (50 $\mathrm{mg} / \mathrm{kg}$ ) i.v. on day 3 after infection, and treatment $(15 \mathrm{mg} / \mathrm{kg}$ i.v.) was then administered daily on days 4, 5, and 6 after infection. Heparin sodium (100 U/kg) was administered i.v. on day 3 after infection, and treatment (100 U/kg i.v.) was maintained daily for 3 days.

Immunohistology and confocal microscopy. Tissues were snapfrozen or fixed in $4 \%$ formaldehyde immediately upon removal. Paraffin-embedded tissue sections were stained by H\&E. Frozen tissue sections were stained by IHC to detect CD3 (145-2C11, BD Biosciences - Pharmingen), F4/80 (Cl:A3:1, AbD Serotec), CD11c (N418, AbD Serotec), Ly6C (AL-21, BD Biosciences), Ly6G (RB6-8C5, eBioscience), CD105 (MJ7/18, BD Biosciences), TF (polyclonal; AF3178, R\&D Systems), and podoplanin (eBio8.1.1, eBioscience), as described previously (73). IHC was performed in Tris buffer ( $\mathrm{pH}$ 7.6). Primary and secondary antibodies were added for 60 and 45 minutes, respectively, at room temperature. Biotin- or horseradish peroxidase-conjugated secondary antibodies were developed using alkaline phosphatase (ABComplex, Vector Laboratories) and levamisole with napthol AS-MX phosphate and Fast Blue BB salt or 3,3'-diaminobenzidine tetrahydrochloride, respectively. Slides were mounted in glycerol and images acquired at $\times 20$ magnification using a Leica CTR6000 microscope (Leica) with a QImaging MicroPublisher 5.0 RTV camera in conjunction with ImageJ (http://imagej.nih.gov/ij/) and QCapture software. Low magnification images were acquired by a Carl Zeiss AxioScan.Z1 Slide Scanner using a 3CCD color 2MP Hitachi $1200 \times 1600$ HV-F202SCL camera. Images were analyzed using Zen blue (edition 2012) slide scan software.

Fluorescent confocal microscopy was performed on frozen liver sections stained with the following: CD41 (clone MWReg30), CD45.2 (clone 104), CD248 (a gift from Clare Isacke, Institute of Cancer Research, London, United Kingdom), CD31 (clone 390) vWF (clone H-300), mouse anti-human fibrin (clone 59D8) (a gift from Rodney Camire, Children's Hospital of Philadelphia, Philadelphia, Pennsylvania, USA) (74), and podoplanin (clone eBio8.1.1) as previously described (73). TF was detected using either a goat anti-mouse antibody (AF3178 from R\&D Systems) or a rat anti-mouse monoclonal antibody (1H1) (75). Staining was performed in PBS plus 1\% FCS. Sections were incubated with primary and secondary antibodies for 90 and 45 minutes, respectively, at room temperature in the dark. Nuclei were detected by Hoechst $33342(10 \mu \mathrm{g} / \mathrm{ml}$ for 2 minutes at room temperature). Slides were mounted using Prolong Gold Anti-Fade Reagent (Invitrogen), and images were taken using a $\times 63$ magnification objective on a LSM510 laser scanning confocal microscope with a Zeiss AxioVert 100M in conjunction with Zeiss LSM image software.

Histological quantification. The proportion of liver area occupied by inflammatory lesions was quantified by point counting (68). The proportion of grid intercepts on lesions was calculated from the total intercepts covering the area counted (60 grids per tissue, representative of the entire tissue section). The percentage of vessel occlusion was measured by the proportion of grid intercepts on thrombi in large vessels out of total grid intercepts on large vessels. For standardization, only the largest vessels (greater than 50 intercepts) were included, and livers were sectioned to similar positions in each tissue.

Flow cytometry. Leukocytes were isolated from livers by collagenase digestion and gradient centrifugation as described elsewhere (76-78). Briefly, livers were mechanically disrupted and immersed in collagenase $\mathrm{D}(1 \mu \mathrm{g} / \mathrm{ml})\left(20\right.$ minutes, $37^{\circ} \mathrm{C}$ with agitation [180 rpm]); reactions 
were stopped with 0.5 mM EDTA. Livers were homogenized through a $70-\mu$ mylon cell strainer in RPMI supplemented with $2 \%$ FBS and $5 \mathrm{mM}$ EDTA. Leukocytes were isolated using a Ficoll Paque PLUS (GE Healthcare) gradient at $375 \mathrm{~g}$ for 20 minutes without brake. Cells were collected from the interface layer, and CD32/Fc $\gamma I I I$ and CD16/Fc $\gamma I I$ receptors were blocked prior to staining with the following antibodies: CD45 (30F11), F4/80 (BM8), CD11c (HL3), CD11b (M1/70), Ly6C (HK1.4), Ly6G (RB6-8C5), NK1.1 (PK136), and podoplanin (eBio8.1.1). IgG isotype control $\mathrm{PE}$ antibody was used in conjunction with anti-podoplanin staining. All antibodies used were conjugated to either FITC, PE, Per-CP-Cy5.5, PE-Cy5, APC, Pacific blue, PE-Cy7, APC, APC-Cy7, biotin, or PE-Texas red; biotinylated antibodies were detected using streptavidin-APC-Сy7. All antibodies were obtained from eBioscience; CD11c-PE-Cy7 was obtained from BD Biosciences. Cells were fixed in 0.1\% paraformaldehyde and were acquired using a CyAn FACS Analyzer (Dako).

Cytokine ELISA. Leukocytes from livers were cultured for 48 hours in RPMI supplemented with $10 \%$ FCS and 1\% penicillin/streptomycin at $37^{\circ} \mathrm{C}$ with $5 \% \mathrm{CO}_{2}$. Supernatants were collected, and IFN- $\gamma$ concentration was determined by ELISA (Ready-Set-Go Kit, eBioscience) according to the manufacturer's instructions. Briefly, wells were coated overnight at $4^{\circ} \mathrm{C}$ with anti-IFN- $\gamma$ capture antibody before samples/standards were added overnight at $4^{\circ} \mathrm{C}$. Biotinylated anti-IFN- $\gamma$ detection antibody was added for 1 hour, and IFN- $\gamma$ signal was detected using avidin-HRP and 3,3',5,5'-tetramethylbenzidine solution. The reaction was stopped with $1 \mathrm{M} \mathrm{H}_{3} \mathrm{PO}_{4}$, and absorbance at $\mathrm{OD} \lambda_{450} \mathrm{~nm}$ was determined using an EMax microplate spectrophotometer (Molecular Devices).

Thrombopoietin serum ELISA. Serum was isolated from whole blood (centrifugation at $6000 \mathrm{~g}$ for 15 minutes) and was stored at $-20^{\circ} \mathrm{C}$. Thrombopoietin concentration was determined using the Thrombopoietin Quantikine ELISA Kit (R\&D Systems) according to the manufacturer's instructions.

Biochemical liver injury assays. Serum was isolated from whole blood, and liver-specific enzymes were detected in conjunction with the Biochemistry Department at the Birmingham Women's Hospital, Birmingham, United Kingdom.

Whole blood analysis. Whole blood was obtained by cardiac puncture into EDTA-coated ( $5 \mathrm{mM}$ ) plastic tubes, and cellular parameters were quantitatively measured using an automated ABX Pentra 60 Hematology Analyzer (Horiba ABX Diagnostics), according to the manufacturer's instructions.
Online supplemental material. Additional examples of flow cytometry gating strategies used, representative FACS plots, and further histological images are shown in Supplemental Figures 1-6.

Statistics. Statistical significance was determined using the 2-tailed Mann-Whitney nonparametric sum of ranks test, 1-way ANOVA with the Dunnett's test, or 2-way ANOVA with Bonferroni's post-test. All $P$ values were calculated using GraphPad Prism version 4.0 and were interpreted as significant at $P \leq 0.05$.

Study approval. All animal procedures were carried out in strict accordance with local ethical approval from the University of Birmingham and the UK Home Office license (project license 30/2850) as covered by the Animals (Scientific Procedures) Act 1986.

\section{Author contributions}

JRH designed and performed experiments and cowrote the manuscript. CNC, SB, EAR, AFL, KL, and CCDM assisted with conducting experiments. SL generated and maintained mouse colonies. MK, SH, GER, CDB, NDJ, IRH, and DHA provided intellectual input. MCG, MC, TJM, AM, NVR, and DK provided reagents. SPW and AFC designed experiments and cowrote the manuscript.

\section{Acknowledgments}

The authors wish to thank the staff of the BMSU and the Biochemistry Department, Birmingham Women's Hospital, for their assistance. We are grateful to Ian MacLennan, Jennifer Marshall, and Alex Brill (University of Birmingham) and Thomas Butler (Ross University) for helpful discussions. This work was supported by grants from the Medical Research Council (G0701275), the Biotechnology and Biological Sciences Research Council (BB/ F022778/1), and the Wellcome Trust (090244) to A.F. Cunningham; and the Wellcome Trust (088410) and the British Heart Foundation (RG/13/18/30563) to S.P. Watson. J.R. Hitchcock was supported by an MRC Studentship to A.F. Cunningham. S.P. Watson is a BHF Chair $(\mathrm{CH} / 03 / 003)$.

Address correspondence to: Adam F. Cunningham or Steve P. Watson, School of Immunity and Infection, MRC Centre for Immune Regulation, University of Birmingham, Edgbaston, Birmingham, B15 2TT, United Kingdom. Phone: 44.121.414.4068; E-mail: a.f.cunningham@bham.ac.uk (A.F. Cunningham), s.p.watson@ bham.ac.uk (S.P. Watson).
1. Monack DM, Mueller A, Falkow S. Persistent bacterial infections: the interface of the pathogen and the host immune system. Nat Rev Microbiol. 2004;2(9):747-765.

2. Richter-Dahlfors A, Buchan AM, Finlay BB. Murine salmonellosis studied by confocal microscopy: Salmonella typhimurium resides intracellularly inside macrophages and exerts a cytotoxic effect on phagocytes in vivo. J Exp Med. 1997;186(4):569-580.

3. Butler T, Bell WR, Levin J, Linh NN, Arnold K. Typhoid fever. Arch Intern Med. 1978;138(3):407-410.

4. Hornick RB, Greisman SE, Woodward TE, DuPont HL, Dawkins AT, Snyder MJ. Typhoid fever: pathogenesis and immunologic control. N Engl J Med. 1970;283(14):739-746.
5. Jones BD, Falkow S. Salmonellosis: host immune responses and bacterial virulence determinants. Annu Rev Immunol. 1996;14:533-561.

6. Huckstep RL. Typhoid Fever And Other Salmonella Infections. London, United Kingdom: Livingstone; 1962.

7. Wright AE, Knapp HH. A note on the causation and treatment of thrombosis occurring in connection with typhoid fever. Med Chir Trans. 1903;86:1-19.

8. Butler T, Knight J, Nath SK, Speelman P, Roy SK, Azad MA. Typhoid fever complicated by intestinal perforation: a persisting fatal disease requiring surgical management. Rev Infect Dis. 1985;7(2):244-256.

9. Brown DE, McCoy MW, Pilonieta MC, Nix RN, Detweiler CS. Chronic murine typhoid fever is a natural model of secondary hemophagocytic lymphohistiocytosis. PLoS One. 2010;5(2):e9441.

10. Vidal S, et al. The Ity/Lsh/Bcg locus: natural resistance to infection with intracellular parasites is abrogated by disruption of the Nramp1 gene. JExp Med.1995;182(3):655-666.

11. Bohme DH, Schneider HA, Lee JM. Some physiopathological parameters of natural resistance to infection in murine salmonellosis. J Exp Med. 1959;110(1):9-26.

12. Cunningham AF, et al. Salmonella induces a switched antibody response without germinal centers that impedes the extracellular spread of infection. JImmunol. 2007;178(10):6200-6207.

13. Mastroeni P, Skepper JN, Hormaeche CE. Effect of anti-tumor necrosis factor $\alpha$ antibodies on histopathology of primary Salmonella infections. 
Infect Immun. 1995;63(9):3674-3682.

14. Engelmann B, Massberg S. Thrombosis as an intravascular effector of innate immunity. Nat Rev Immunol. 2013;13(1):34-45.

15. Sun $\mathrm{H}$, et al. Plasminogen is a critical host pathogenicity factor for group A streptococcal infection. Science. 2004;305(5688):1283-1286.

16. Massberg S, et al. Reciprocal coupling of coagulation and innate immunity via neutrophil serine proteases. Nat Med.2010;16(8):887-896.

17. Clark SR, et al. Platelet TLR4 activates neutrophil extracellular traps to ensnare bacteria in septic blood. Nat Med. 2007;13(4):463-469.

18. Cheng AG, McAdow M, Kim HK, Bae T, Missiakas DM, Schneewind O. Contribution of coagulases towards Staphylococcus aureus disease and protective immunity. PLoS Pathog. 2010;6(8):e1001036.

19. Arman M, et al. Amplification of bacteriainduced platelet activation is triggered by Fc $\gamma$ RIIA, integrin $\alpha \operatorname{IIb} \beta 3$, and platelet factor 4 . Blood. 2014;123(20):3166-3174.

20. Tilley DO, et al. Glycoprotein Ib $\alpha$ and Fc $\gamma$ RIIa play key roles in platelet activation by the colonizing bacterium, Streptococcus oralis. J Thromb Haemost. 2013;11(5):941-950.

21. Cox D, Kerrigan SW, Watson SP. Platelets and the innate immune system: mechanisms of bacterialinduced platelet activation. J Thromb Haemost. 2011;9(6):1097-1107.

22. Suzuki-Inoue K, et al. A novel Syk-dependent mechanism of platelet activation by the C-type lectin receptor CLEC-2. Blood. 2006;107(2):542-549.

23. Astarita JL, Acton SE, Turley SJ. Podoplanin: emerging functions in development, the immune system, and cancer. Front Immunol. 2012;3:283.

24. Hou TZ, et al. A distinct subset of podoplanin (gp38) expressing F4/80+ macrophages mediate phagocytosis and are induced following zymosan peritonitis. FEBS Lett. 2010;584(18):3955-3961.

25. Jackson A, Nanton MR, O'Donnell H, Akue AD, McSorley SJ. Innate immune activation during Salmonella infection initiates extramedullary erythropoiesis and splenomegaly. J Immunol. 2010;185(10):6198-6204.

26. Gibbins JM, Okuma M, Farndale R, Barnes M, Watson SP. Glycoprotein VI is the collagen receptor in platelets which underlies tyrosine phosphorylation of the Fc receptor $\gamma$-chain. FEBS Lett. 1997;413(2):255-259.

27. Talbot $S$, et al. Toll-like receptor 4 signalling through MyD88 is essential to control Salmonella enterica serovar typhimurium infection, but not for the initiation of bacterial clearance. Immunology. 2009;128(4):472-483.

28. Schwabe RF, Seki E, Brenner DA. Toll-like receptor signaling in the liver. Gastroenterology. 2006;130(6):1886-1900.

29. Bao S, Beagley KW, France MP, Shen J, Husband AJ. Interferon- $\gamma$ plays a critical role in intestinal immunity against Salmonella typhimurium infection. Immunology. 2000;99(3):464-472.

30. Suzuki-Inoue K, et al. Involvement of the snake toxin receptor CLEC-2, in podoplanin-mediated platelet activation, by cancer cells. J Biol Chem . 2007;282(36):25993-26001.

31. Christou CM, et al. Renal cells activate the platelet receptor CLEC-2 through podoplanin.
Biochem J. 2008;411(1):133-140.

32. Acton SE, et al. Podoplanin-rich stromal networks induce dendritic cell motility via activation of the C-type lectin receptor CLEC-2. Immunity. 2012;37(2):276-289.

33. Boulaftali $Y$, et al. Platelet ITAM signaling is critical for vascular integrity in inflammation. J Clin Invest. 2013;123(2):908-916.

34. Benezech C, et al. CLEC-2 is required for development and maintenance of lymph nodes. Blood. 2014;123(20):3200-3207.

35. Guha M, Mackman N. LPS induction of gene expression in human monocytes. Cell Signal. 2001;13(2):85-94.

36. VanCott JL, et al. Regulation of host immune responses by modification of Salmonella virulence genes. Nat Med. 1998;4(11):1247-1252.

37. Vazquez-Torres A, et al. Toll-like receptor 4 dependence of innate and adaptive immunity to Salmonella: importance of the Kupffer cell network. JImmunol. 2004;172(10):6202-6208.

38. Changming W, et al. Monocytes can be induced to express lymphatic phenotypes. Lymphology. 2011;44(2):48-53.

39. Lax S, et al. CD248/Endosialin is dynamically expressed on a subset of stromal cells during lymphoid tissue development, splenic remodeling and repair. FEBS Lett. 2007;581(18):3550-3556.

40. Zarychanski $\mathrm{R}$, et al. The efficacy and safety of heparin in patients with sepsis: a systematic review and metaanalysis. Crit Care Med. 2015;43(3):511-518.

41. Finney BA, et al. CLEC-2 and Syk in the megakaryocytic/platelet lineage are essential for development. Blood. 2012;119(7):1747-1756.

42. Hughes CE, Navarro-Nunez L, Finney BA, Mourao-Sa D, Pollitt AY, Watson SP. CLEC-2 is not required for platelet aggregation at arteriolar shear. J Thromb Haemost. 2010;8(10):2328-2332.

43. Suzuki-Inoue $\mathrm{K}$, et al. Essential in vivo roles of the C-type lectin receptor CLEC-2: embryonic/ neonatal lethality of CLEC-2-deficient mice by blood/lymphatic misconnections and impaired thrombus formation of CLEC-2-deficient platelets. J Biol Chem. 2010;285(32):24494-24507.

44. Bender M, et al. Combined in vivo depletion of glycoprotein VI and C-type lectin-like receptor 2 severely compromises hemostasis and abrogates arterial thrombosis in mice. Arterioscler Thromb Vasc Biol. 2013;33(5):926-934.

45. Waddington CS, et al. An outpatient, ambulantdesign, controlled human infection model using escalating doses of Salmonella Typhi challenge delivered in sodium bicarbonate solution. Clin Infect Dis. 2014;58(9):1230-1240.

46. Gordon MA, et al. Invasive non-typhoid salmonellae establish systemic intracellular infection in HIV-infected adults: an emerging disease pathogenesis. Clin Infect Dis. 2010;50(7):953-962.

47. Canvin JR, et al. The role of pneumolysin and autolysin in the pathology of pneumonia and septicemia in mice infected with a type 2 pneumococcus. J Infect Dis. 1995;172(1):119-123.

48. Sutton AC, Sevier CE. A study of the bacteriaemia in lobar pneumonia. Bull Johns Hopkins Hosp. 1917;28:315-318.

49. Nurden AT. Platelets, inflammation and tissue regeneration. Thromb Haemost. 2011;105(suppl 1):S13-S33.

50. Azad AK, Islam R, Salam MA, Alam AN, Islam $\mathrm{M}$, Butler T. Comparison of clinical features and pathologic findings in fatal cases of typhoid fever during the initial and later stages of the disease. Am J Trop Med Hyg. 1997;56(5):490-493.

51. Cohen JI, Bartlett JA, Corey GR. Extra-intestinal manifestations of salmonella infections. Medicine (Baltimore). 1987;66(5):349-388.

52. Govoni G, Gros P. Macrophage NRAMP1 and its role in resistance to microbial infections. Inflamm Res. 1998;47(7):277-284.

53. Noris M, Mescia F, Remuzzi G. STEC-HUS, atypical HUS and TTP are all diseases of complement activation. Nat Rev Nephrol. 2012;8(11):622-633.

54. Ross EA, et al. Resolving Salmonella infection reveals dynamic and persisting changes in murine bone marrow progenitor cell phenotype and function. Eur J Immunol. 2014;44(8):2318-2330.

55. Seebaran AR, Coovadia YM, Bhana RH, Rajput MC, Naidoo BT, Haffejee IE. Typhoid fever in the adult and paediatric Indian population of Durban. S Afr Med J. 1990;77(1):14-17.

56. Toso JF, et al. Phase I study of the intravenous administration of attenuated Salmonella typhimurium to patients with metastatic melanoma. JClin Oncol. 2002;20(1):142-152.

57. Jayachandran M, Miller VM, Brunn GJ, Owen WG. Platelet response as a sentinel marker of toll-like receptor 4 activation in mice. Thromb Res. 2010;126(5):414-417.

58. Gondwe EN, et al. Importance of antibody and complement for oxidative burst and killing of invasive nontyphoidal Salmonella by blood cells in Africans. Proc Natl Acad Sci U S A. 2010;107(7):3070-3075.

59. Graham SM, Molyneux EM, Walsh AL, Cheesbrough JS, Molyneux ME, Hart CA. Nontyphoidal Salmonella infections of children in tropical Africa. Pediatr Infect Dis J. 2000;19(12):1189-1196.

60. MacLennan CA, et al. The neglected role of antibody in protection against bacteremia caused by nontyphoidal strains of Salmonella in African children. JClin Invest. 2008;118(4):1553-1562.

61. Usmani GN, Woda BA, Newburger PE. Advances in understanding the pathogenesis of HLH. $\mathrm{Br} \mathrm{J}$ Haematol. 2013;161(5):609-622.

62. Dalton DK, Pitts-Meek S, Keshav S, Figari IS, Bradley A, Stewart TA. Multiple defects of immune cell function in mice with disrupted interferon-gamma genes. Science. 1993;259(5102):1739-1742.

63. Mombaerts P, Iacomini J, Johnson RS, Herrup K, Tonegawa S, Papaioannou VE. RAG-1-deficient mice have no mature B and T lymphocytes. Cell. 1992;68(5):869-877.

64. Reinhardt RL, Liang HE, Locksley RM. Cytokinesecreting follicular $\mathrm{T}$ cells shape the antibody repertoire. Nat Immunol. 2009;10(4):385-393.

65. Stadtfeld M, Graf T. Assessing the role of hematopoietic plasticity for endothelial and hepatocyte development by non-invasive lineage tracing. Development. 2005;132(1):203-213.

66. Kato K, et al. The contribution of glycoprotein VI to stable platelet adhesion and thrombus formation illustrated by targeted gene deletion. Blood. 2003;102(5):1701-1707.

67. Mendiratta SK, Martin WD, Hong S, Boesteanu 


\section{RESEARCH ARTICLE}

A, Joyce S, Van Kaer L. CD1d1 mutant mice are deficient in natural $\mathrm{T}$ cells that promptly produce IL-4. Immunity. 1997;6(4):469-477.

68. Cunningham AF, et al. Responses to the soluble flagellar protein FliC are Th2, while those to FliC on Salmonella are Th1. Eur JImmunol. 2004;34(11):2986-2995.

69. Buiting AM, Van Rooijen N. Liposome mediated depletion of macrophages: an approach for fundamental studies. J Drug Target. 1994;2(5):357-362.

70. Van Rooijen N. The liposome-mediated macrophage 'suicide' technique. JImmunol Methods. 1989;124(1):1-6.

71. Van Rooijen N, Sanders A. Kupffer cell depletion by liposome-delivered drugs: comparative activ- ity of intracellular clodronate, propamidine, and ethylenediaminetetraacetic acid. Hepatology. 1996;23(5):1239-1243.

72. Wong CH, Jenne CN, Petri B, Chrobok NL, Kubes P. Nucleation of platelets with blood-borne pathogens on Kupffer cells precedes other innate immunity and contributes to bacterial clearance. Nat Immunol. 2013;14(8):785-792.

73. Flores-Langarica A, et al. Systemic flagellin immunization stimulates mucosal $\mathrm{CD}_{103^{+}}$dendritic cells and drives Foxp $3^{+}$regulatory T cell and IgA responses in the mesenteric lymph node. JImmunol. 2012;189(12):5745-5754.

74. Weiler-Guettler $\mathrm{H}$, et al. A targeted point mutation in thrombomodulin generates viable mice with a prethrombotic state. J Clin Invest. 1998;101(9):1983-1991.

75. Kirchhofer D, Moran P, Bullens S, Peale F, Bunting S. A monoclonal antibody that inhibits mouse tissue factor function. J Thromb Haemost. 2005;3(5):1098-1099.

76. Klein I, et al. Kupffer cell heterogeneity: functional properties of bone marrow derived and sessile hepatic macrophages. Blood. 2007;110(12):4077-4085.

77. Li PZ, Li JZ, Li M, Gong JP, He K. An efficient method to isolate and culture mouse Kupffer cells. Immunol Lett. 2013;158(1-2):52-56.

78. Sakai A, Tanaka S, Kountz SL. Liver and immune responses. Transplantation. 1978;25(3):110-114. 